\title{
Synthesis of Esters by in situ Formation and Trapping of
}

Diazoalkanes

Richard A. Squitieri ${ }^{1}$, Galen P. Shearn-Nance ${ }^{1}$, Jason E. Hein ${ }^{2}$, Jared T. Shaw ${ }^{* 1}$

${ }^{1}$ Department of Chemistry, University of California, One Shields Ave, Davis, CA 95616

${ }^{2}$ Department of Chemistry, University of British Columbia, Vancouver, BC V6T 1Z1, Canada

Contents

Page

$\begin{array}{lr}\text { FTIR Experimental } & \text { S-2 }\end{array}$

$\begin{array}{lr}\text { FTIR Data } & \text { S-3 }\end{array}$

$\begin{array}{lr}\text { NMR Spectra } & \text { S-5 }\end{array}$ 


\section{FTIR Experimentals}

\section{General Comments}

In situ FT-IR monitoring was conducted with a ReactIR 15 equipped with a DiComp (Diamond) ATR probe connected via an $\mathrm{AgX}$ (silver halide) $6 \mathrm{~mm} \times 1.5 \mathrm{~m}$ fiber. Reaction temperatures were monitored using an internal thermistor in the IR probe. Sampling was carried out over $2000-800 \mathrm{~cm}^{-1}$ at 4 wavenumber resolution with $1 \mathrm{x}$ gain.

\section{Ester formation (acid last)}

A solution of hydrazone (75 $\mathrm{mg}, 0.38 \mathrm{mmol})$ in anhydrous $\mathrm{CH}_{2} \mathrm{Cl}_{2}(3.2 \mathrm{~mL})$ was prepared in a 7 $\mathrm{mL}$ amber vial fitted with a rubber septum through which the FTIR probe was inserted. The vessel was purged with argon and once a stable FTIR spectrum was observed, $\mathrm{MnO}_{2}(66 \mathrm{mg}$, $0.76 \mathrm{mmol}), \mathrm{KH}_{2} \mathrm{PO}_{4}(104 \mathrm{mg}, 0.764 \mathrm{mmol})$, and finally benzoic acid were added $(70 \mathrm{mg}, 0.57$ mmol). Reaction progress and the buildup of diazocompound were monitored by FTIR using three peaks $\left(1695 \mathrm{~cm}^{-1}, 1499 \mathrm{~cm}^{-1}\right.$, and $\left.1109 \mathrm{~cm}^{-1}\right)$ relative to a single point baseline.

\section{Ester formation (dropwise addition of hydrazone)}

A suspension of $\mathrm{MnO}_{2}$ (66 mg, $\left.0.76 \mathrm{mmol}\right), \mathrm{KH}_{2} \mathrm{PO}_{4}(104 \mathrm{mg}, 0.764 \mathrm{mmol})$, and benzoic acid (70 mg, $0.57 \mathrm{mmol}$ ) in anhydrous $\mathrm{CH}_{2} \mathrm{Cl}_{2}(2.2 \mathrm{~mL}$ ) was prepared in a $7 \mathrm{~mL}$ amber vial fitted with a rubber septum through which the FTIR probe was inserted. The vessel was purged with argon and once a stable FTIR spectrum was observed hydrazone $(75 \mathrm{mg}, 0.38 \mathrm{mmol}$ ) was added dropwise as a solution in $\mathrm{CH}_{2} \mathrm{Cl}_{2}(1.0 \mathrm{~mL})$ via a syringe pump over $10 \mathrm{~min}$. Reaction progress and the buildup of diazocompound were monitored by FTIR using three peaks $\left(1695 \mathrm{~cm}^{-1}, 1499\right.$ $\mathrm{cm}^{-1}$, and $1109 \mathrm{~cm}^{-1}$ ) relative to a single point baseline.

\section{Ester formation ( $\mathrm{MnO}_{2}$ last)}

A suspension of hydrazone (75 mg, $0.38 \mathrm{mmol}), \mathrm{KH}_{2} \mathrm{PO}_{4}(104 \mathrm{mg}, 0.764 \mathrm{mmol})$, and benzoic acid (70 $\mathrm{mg}, 0.57 \mathrm{mmol})$ in anhydrous $\mathrm{CH}_{2} \mathrm{Cl}_{2}(3.2 \mathrm{~mL})$ was prepared in a $7 \mathrm{~mL}$ amber vial fitted with a rubber septum through which the FTIR probe was inserted. The vessel was purged with argon and once a stable FTIR spectrum was observed, $\mathrm{MnO}_{2}(66 \mathrm{mg}, 0.76 \mathrm{mmol}$ ) was added. Reaction progress and the buildup of diazocompound were monitored by FTIR using three peaks $\left(1695 \mathrm{~cm}^{-1}, 1499 \mathrm{~cm}^{-1}\right.$, and $\left.1109 \mathrm{~cm}^{-1}\right)$ relative to a single point baseline. 


\section{FTIR Data}

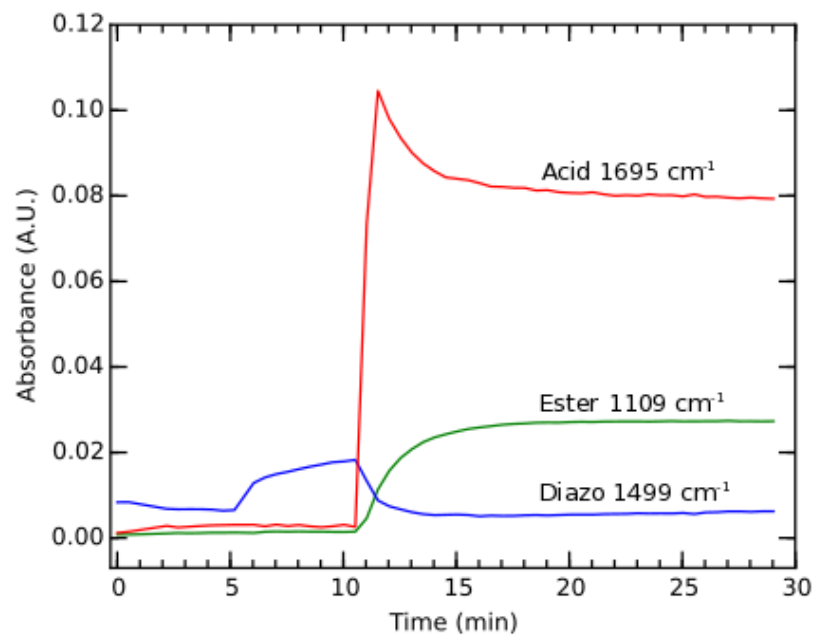

Figure S1. ReactIR data for the "acid last" esterification conditions (Table 1) showing buildup of diphenyldiazomethane (blue), consumption of benzoic acid (red), and appearance of diphenylmethyl benzoate (2, green). Note: the acid carbonyl stretch does not return to baseline because of interference from the ester stretch that grows in.

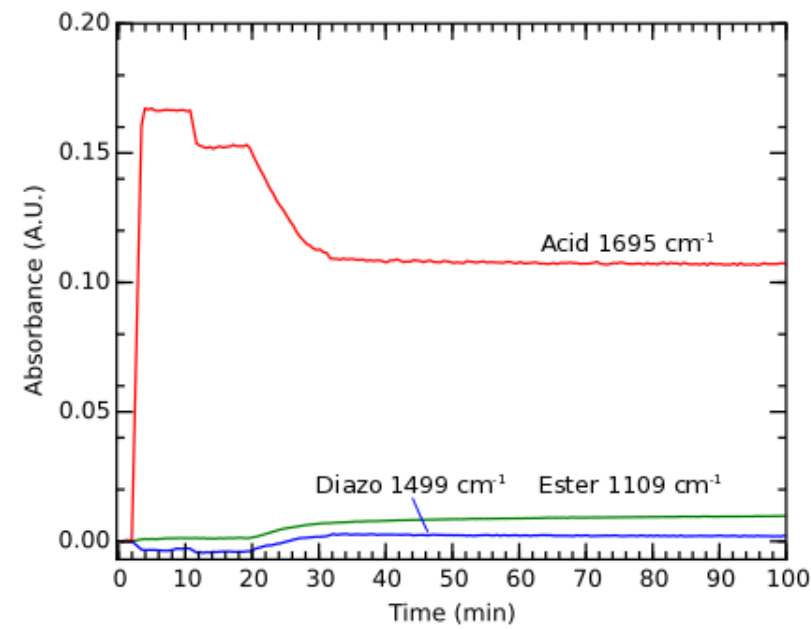

Figure S2. ReactIR data for the "hydrazone last" esterification conditions (Table 1) showing consumption of benzoic acid (red) and appearance of diphenylmethyl benzoate (2, green). Note: $\mathrm{MnO}_{2}$ was added at 10 minutes, followed by addition of benzophenone hydrazone at 19 minutes over a 10 minute period. 


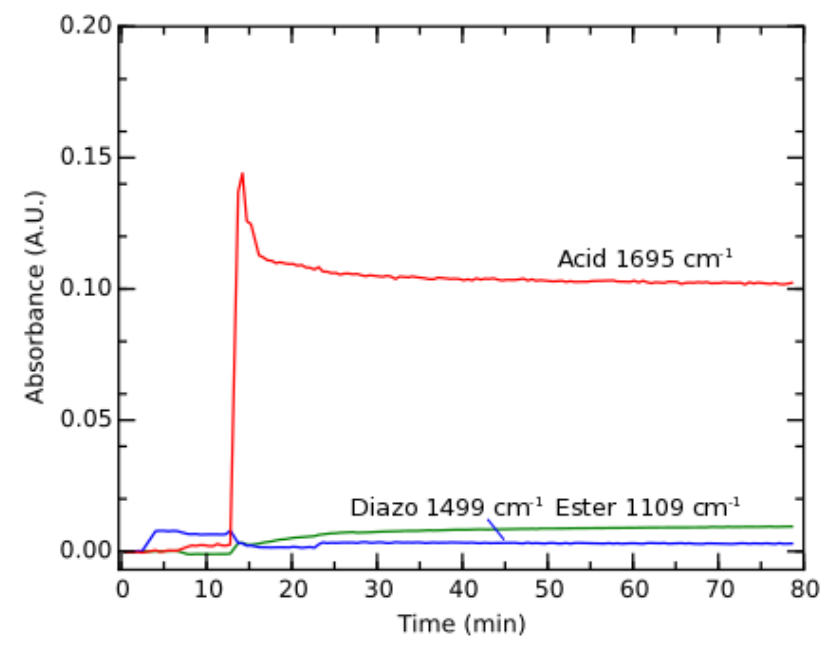

Figure S-3. ReactIR data for the " $\mathrm{MnO}_{2} "$ esterification conditions (See Table 1) consumption of benzoic acid (red), and appearance of diphenylmethyl benzoate (2, green). Note: $\mathrm{MnO}_{2}$ was added at 14 minutes to a solution of benzophenone hydrazone and benzoic acid. 
NMR Data

benzylidenehydrazine (4a) ${ }^{1} \mathrm{H}$ NMR (600 MHz, $\mathrm{CDCl}_{3}$ )

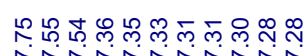

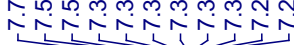

$\stackrel{\substack{0 \\ 0}}{0}$

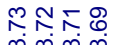
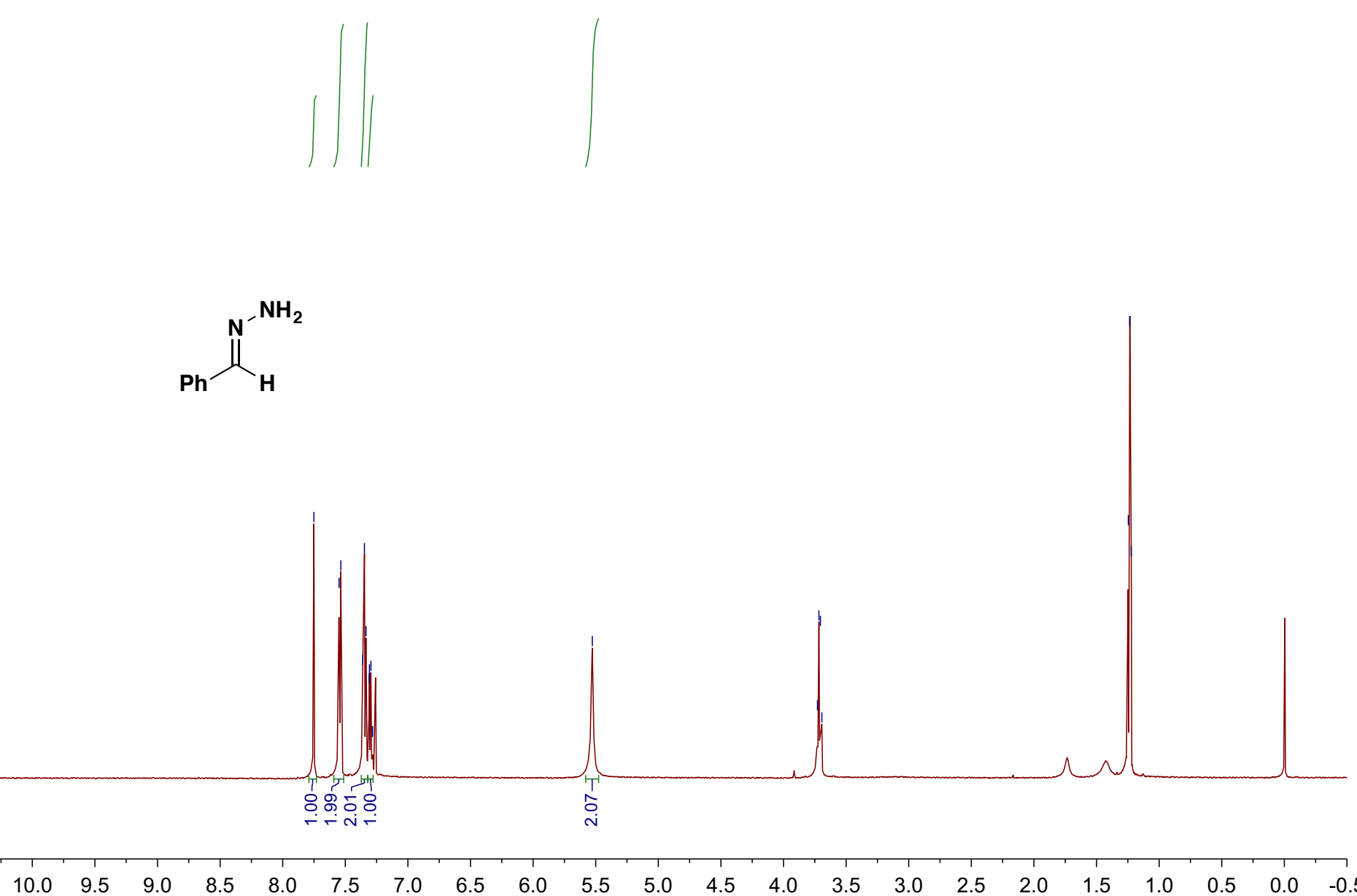
(4-methoxybenzylidene)hydrazine (4b) ${ }^{1} \mathrm{H}$ NMR (600 MHz, $\mathrm{CDCl}_{3}$ )

i㓡
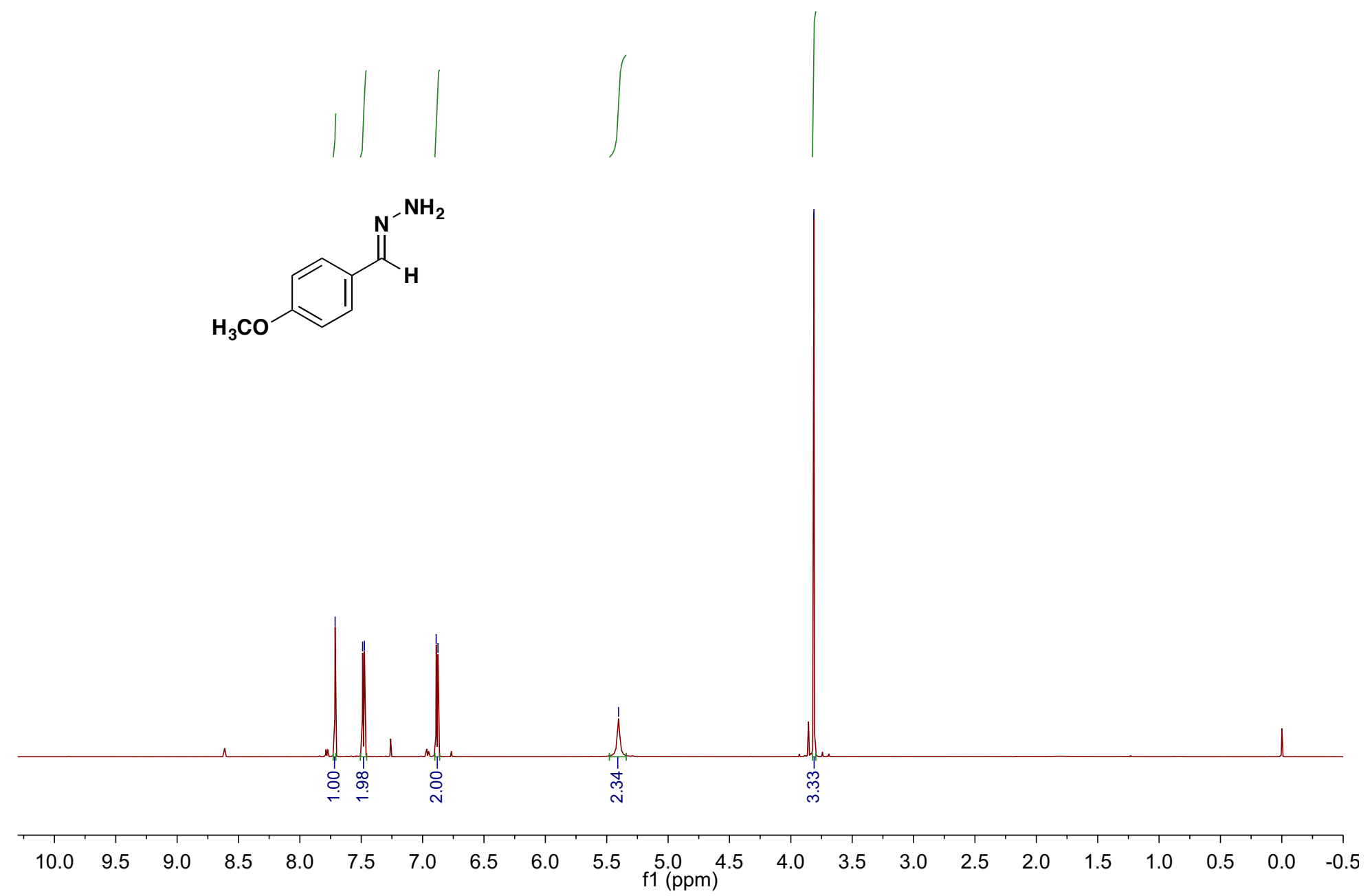
4-(hydrazonomethyl)benzonitrile (4c) ${ }^{1} \mathrm{H}$ NMR (400 $\mathrm{MHz}, \mathrm{CDCl}_{3}$ )
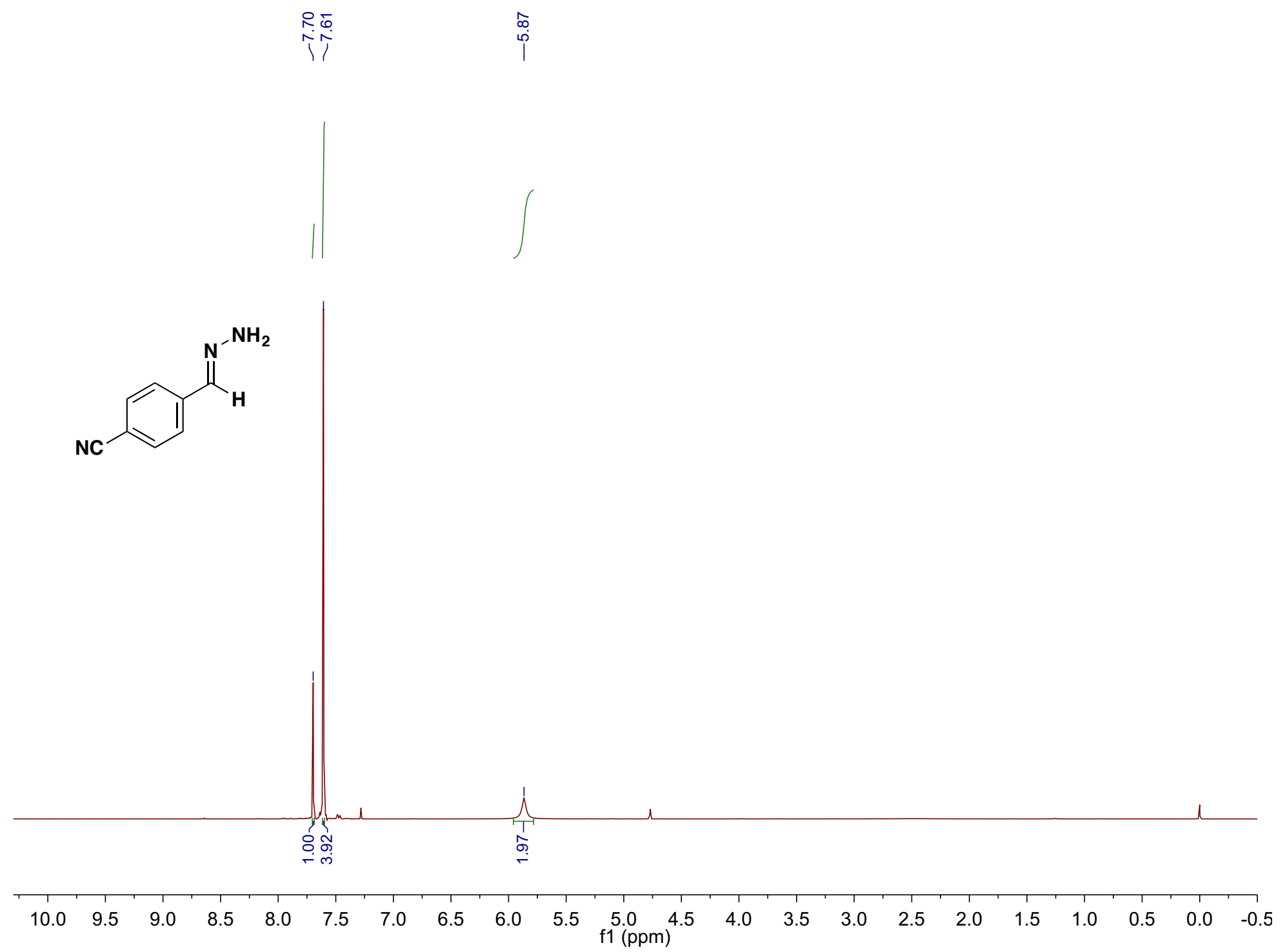
(E)-(2,4,6-trimethylbenzylidene)hydrazine (4d) ${ }^{1} \mathrm{H}$ NMR (400 MHz, $\left.\mathrm{CDCl}_{3}\right)$

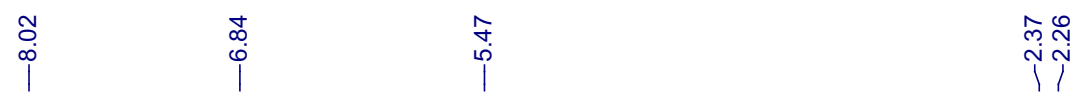

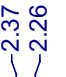
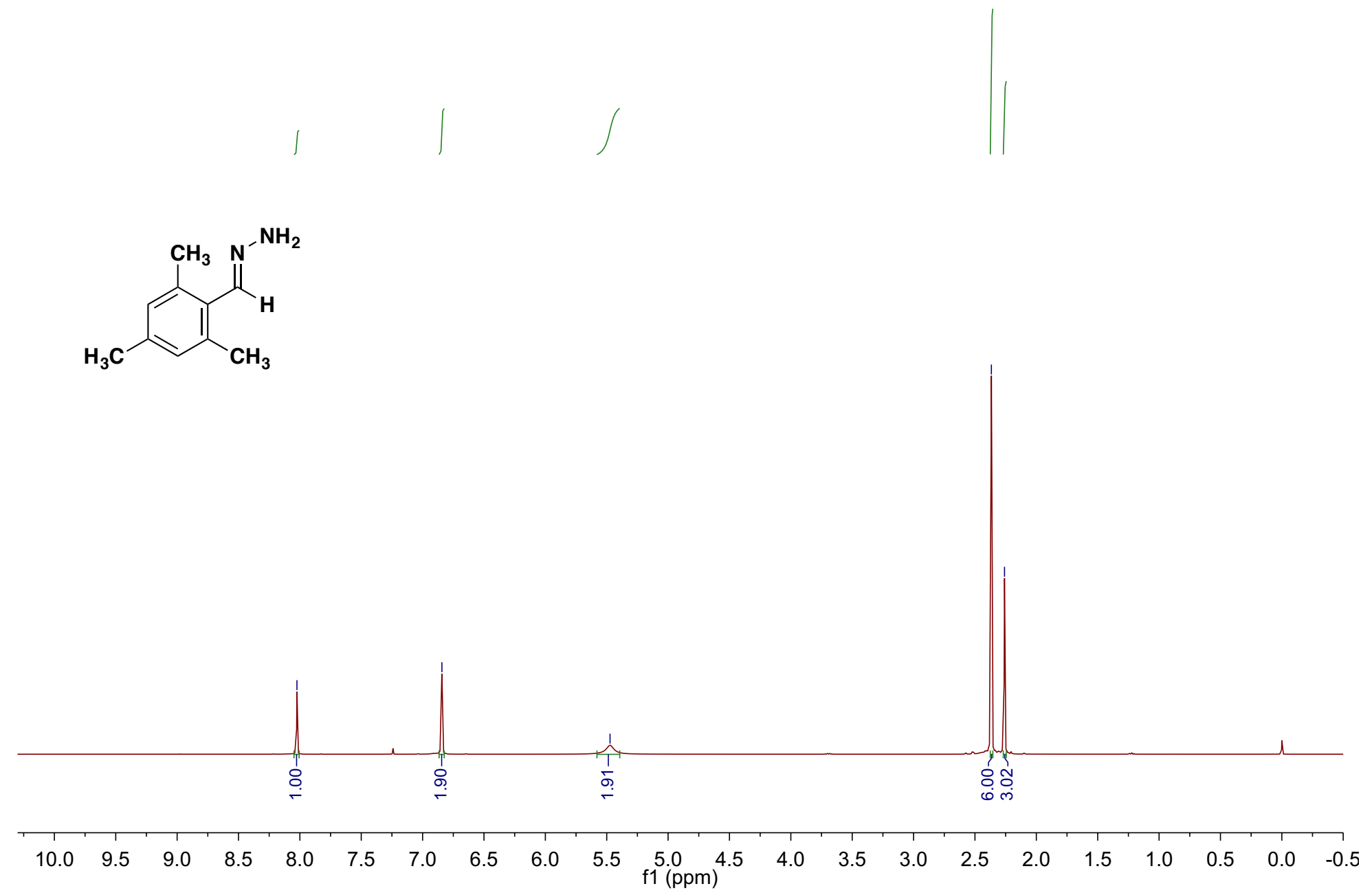
(E)-(2,4,6-trimethylbenzylidene)hydrazine (4d) ${ }^{13} \mathrm{C}$ NMR (100 MHz, $\left.\mathrm{CDCl}_{3}\right)$

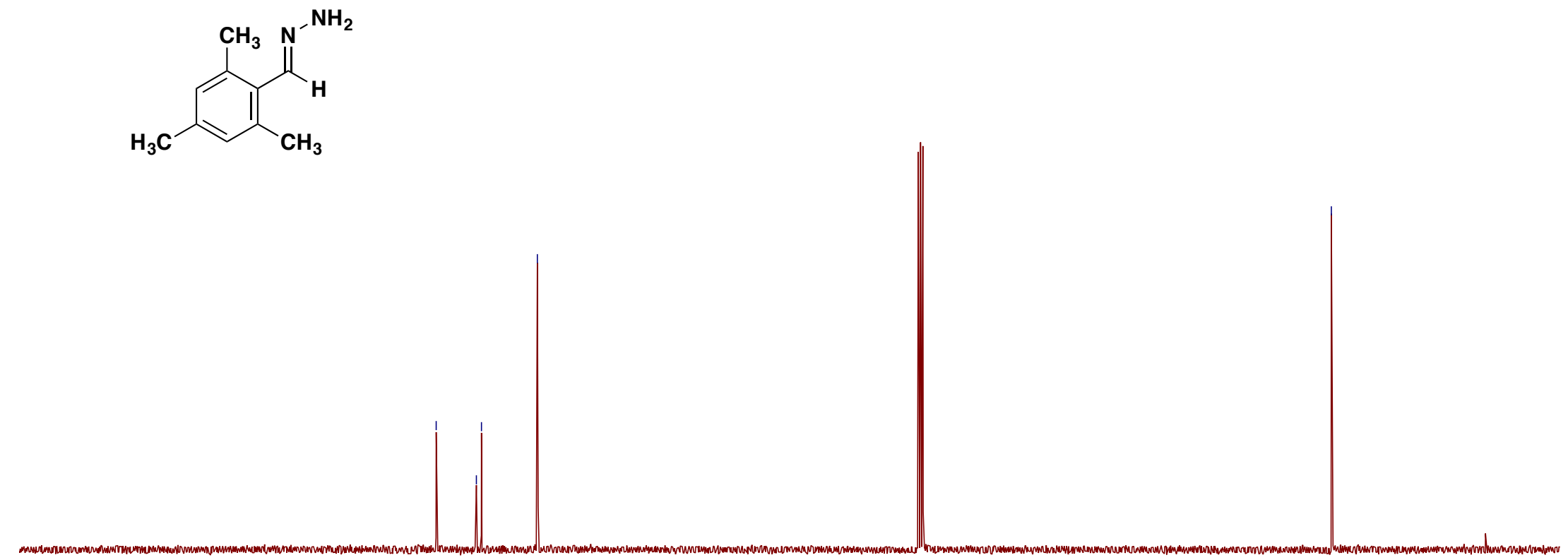

30

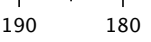

170

160

$150 \quad 140$

$130 \quad 120$

110

100 f1 (ppm) 90

$80 \quad 70$

60

40

(1) 
(3,4-dihydronaphthalen-1(2H)-ylidene)hydrazine (4e) ${ }^{1} \mathrm{H}$ NMR (600 $\left.\mathrm{MHz}, \mathrm{CDCl}_{3}\right)$

붕 유휴

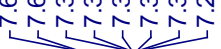

ปั)

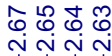

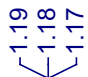
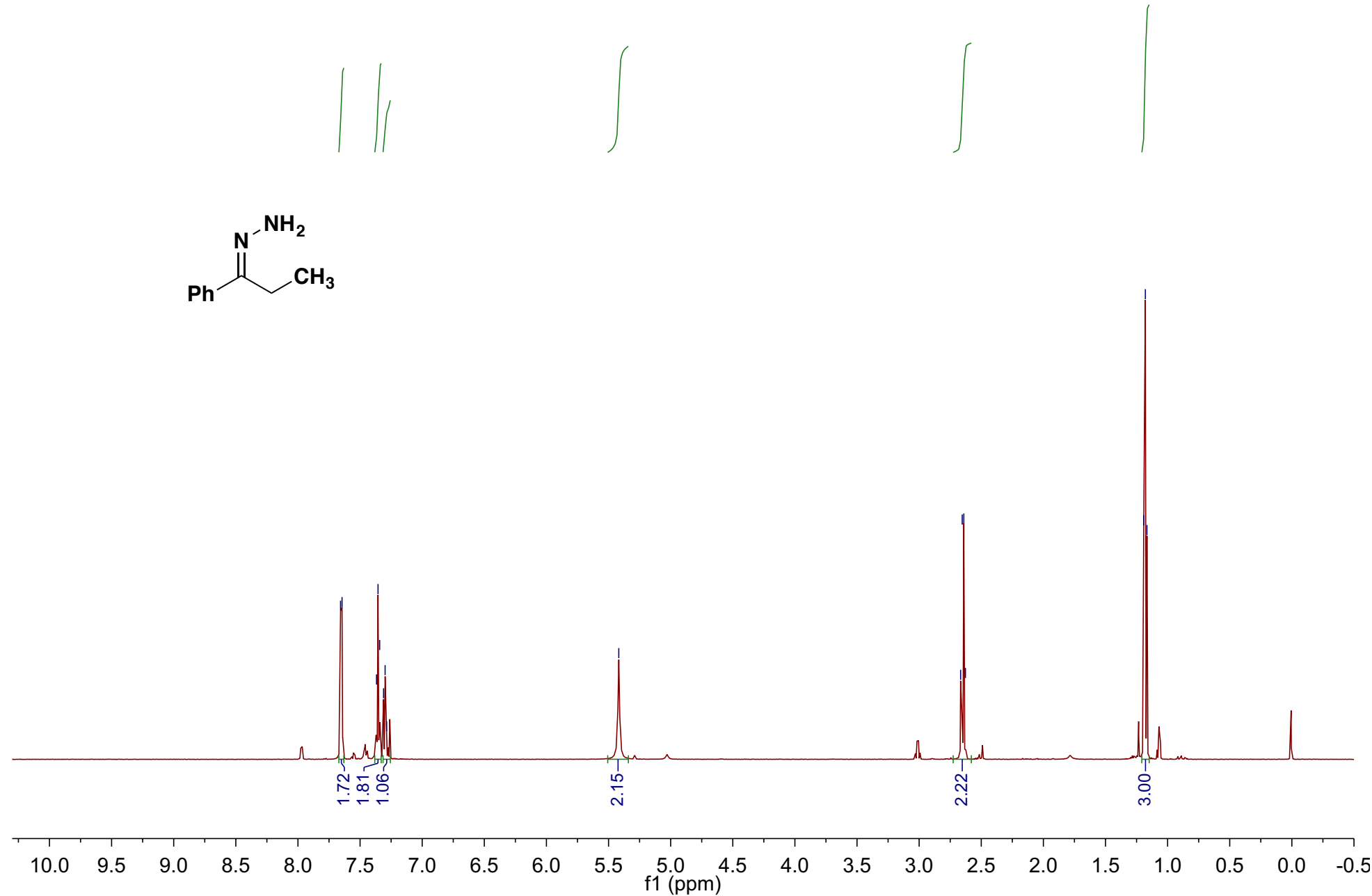
1-phenylethylidene)hydrazine (4f) ${ }^{1} \mathrm{H}$ NMR (600 $\left.\mathrm{MHz} \mathrm{CDCl}_{3}\right)$

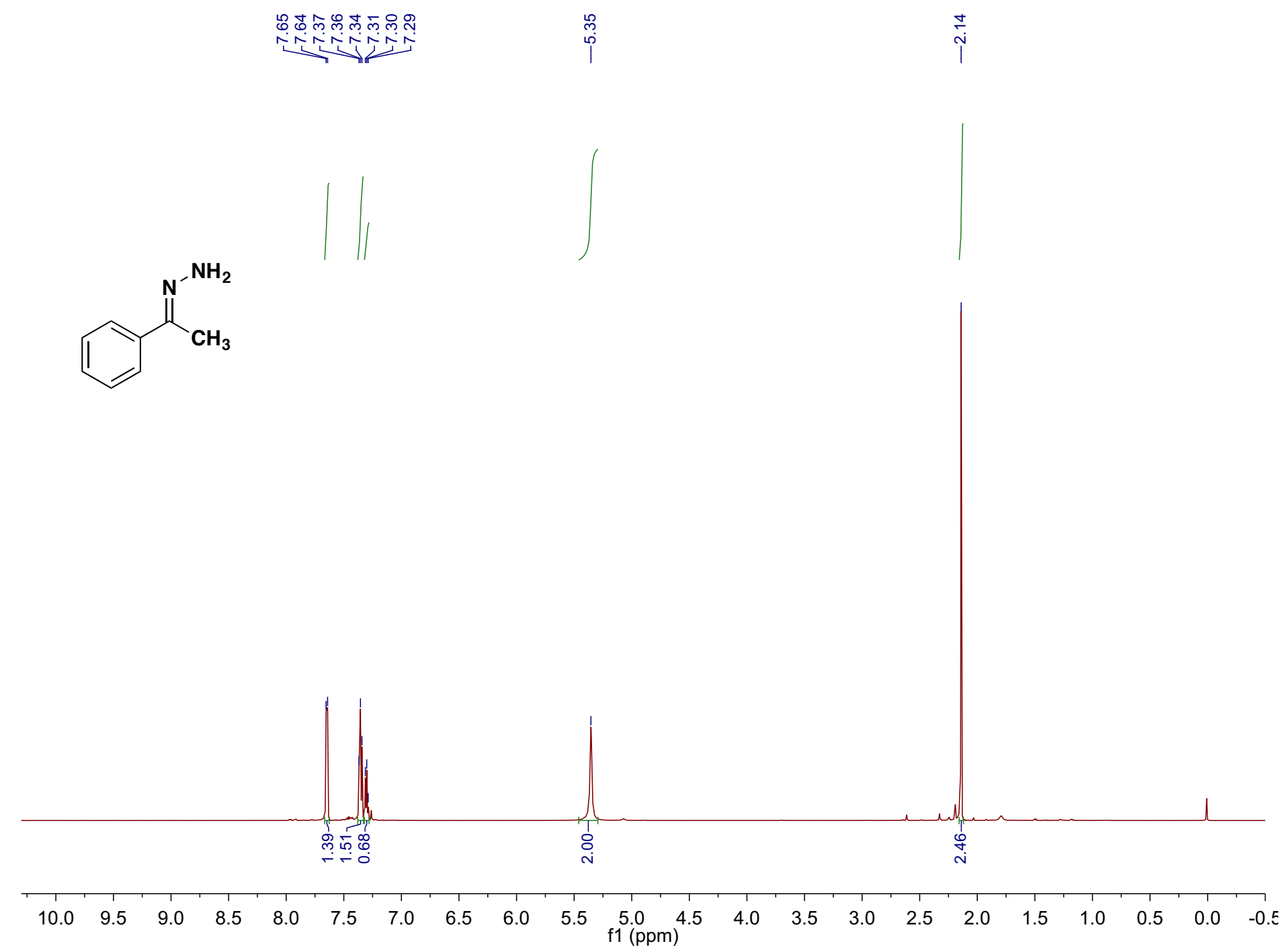


3,4-dihydronaphthalen-1(2H)-ylidene)hydrazine (4g) ${ }^{1} \mathrm{H}$ NMR (600 $\left.\mathrm{MHz} \mathrm{CDCl}_{3}\right)$

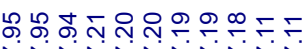

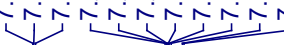

i̊

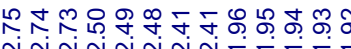

些证

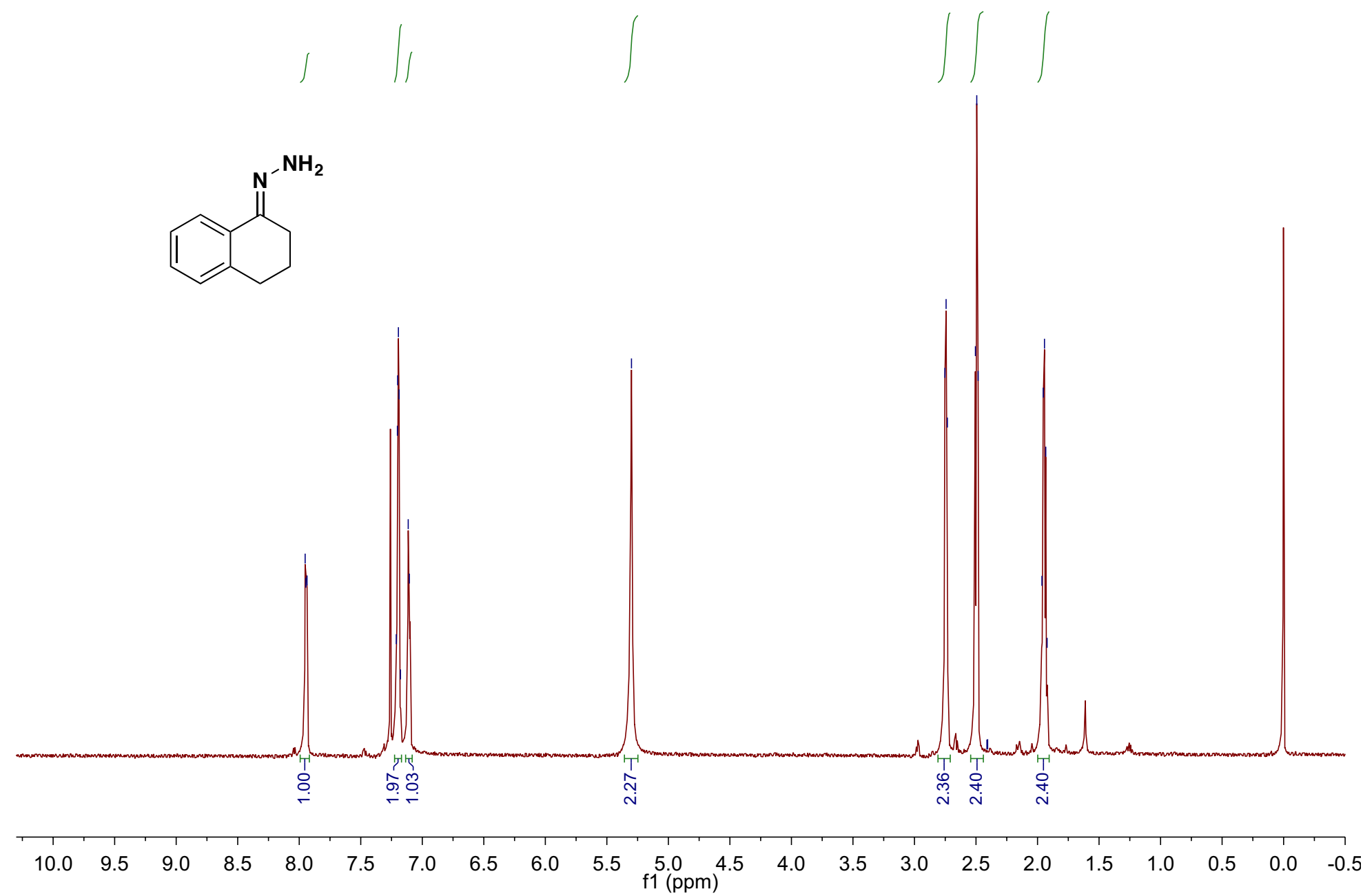


benzhydryl benzoate (2) ${ }^{1} \mathrm{H}$ NMR (600 $\left.\mathrm{MHz}, \mathrm{CDCl}_{3}\right)-$ Method A

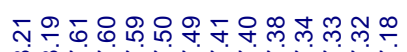

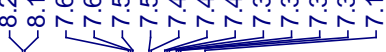

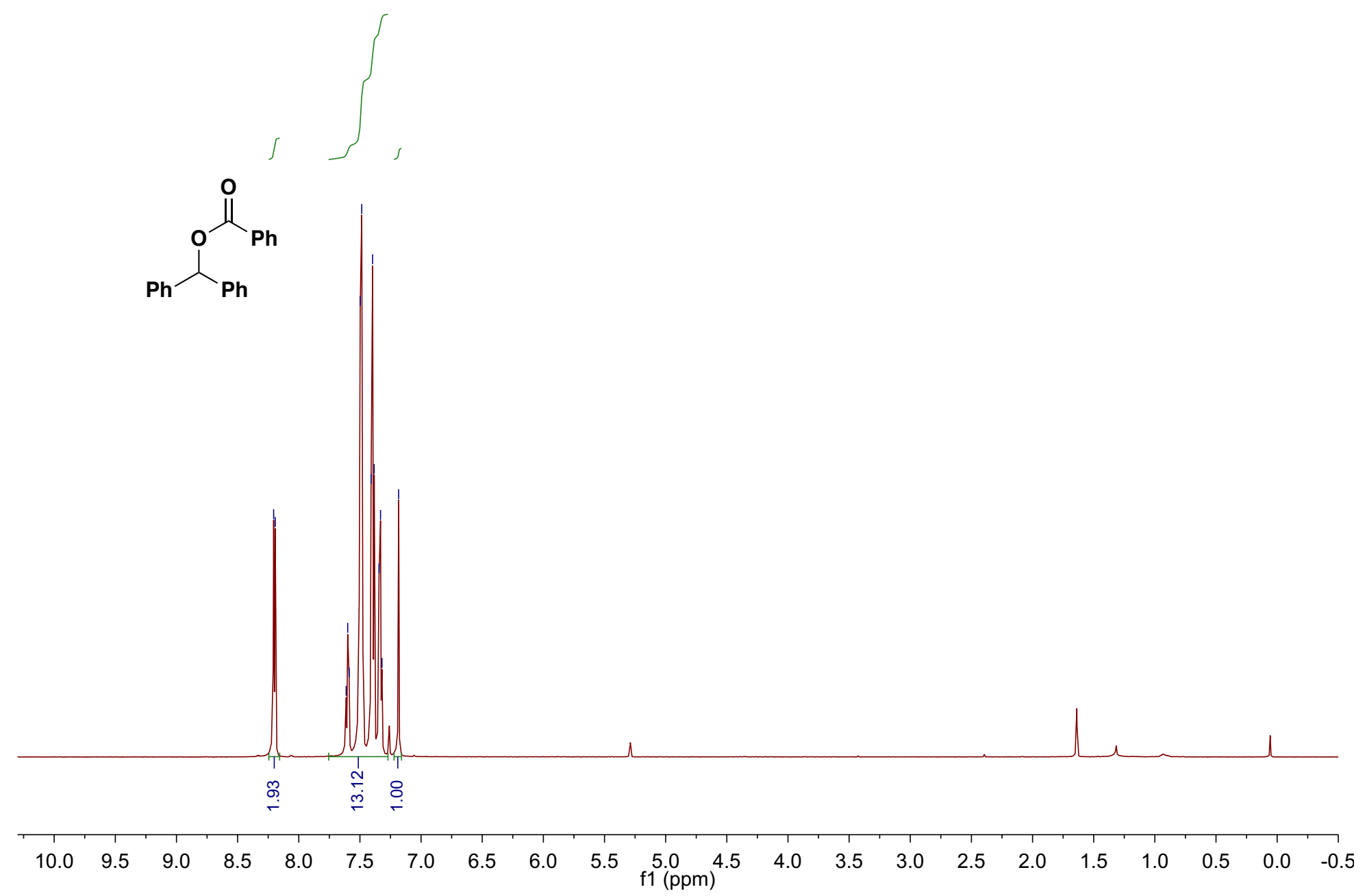


benzhydryl 4-methoxybenzoate (3a) ${ }^{1} \mathrm{H}$ NMR (600 MHz, $\mathrm{CDCl}_{3}$ ) - Method A

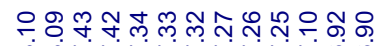

inivinitiog
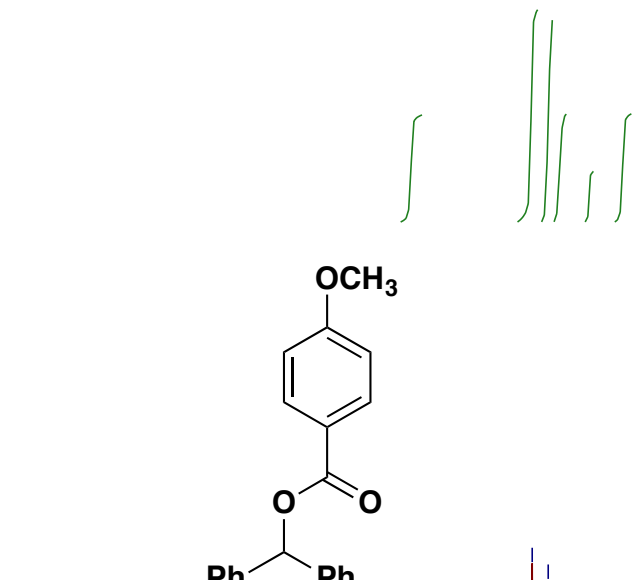

$\mathrm{OCH}_{3}$

$\mathrm{Ph} P \mathrm{Ph}$

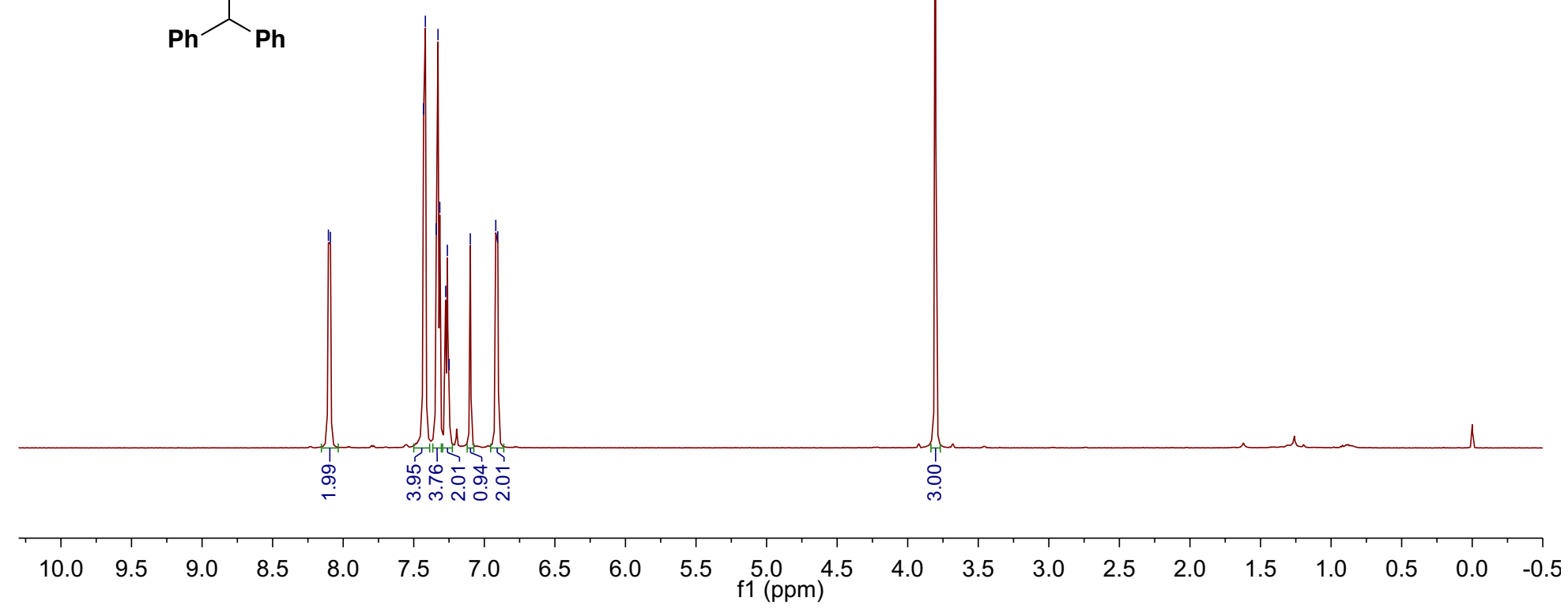


benzhydryl 4-methoxybenzoate (3a) ${ }^{13} \mathrm{C}$ NMR (151 MHz, CDCl $)$

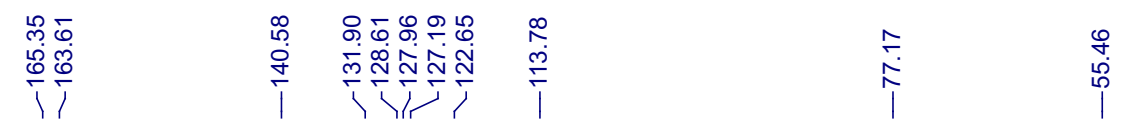

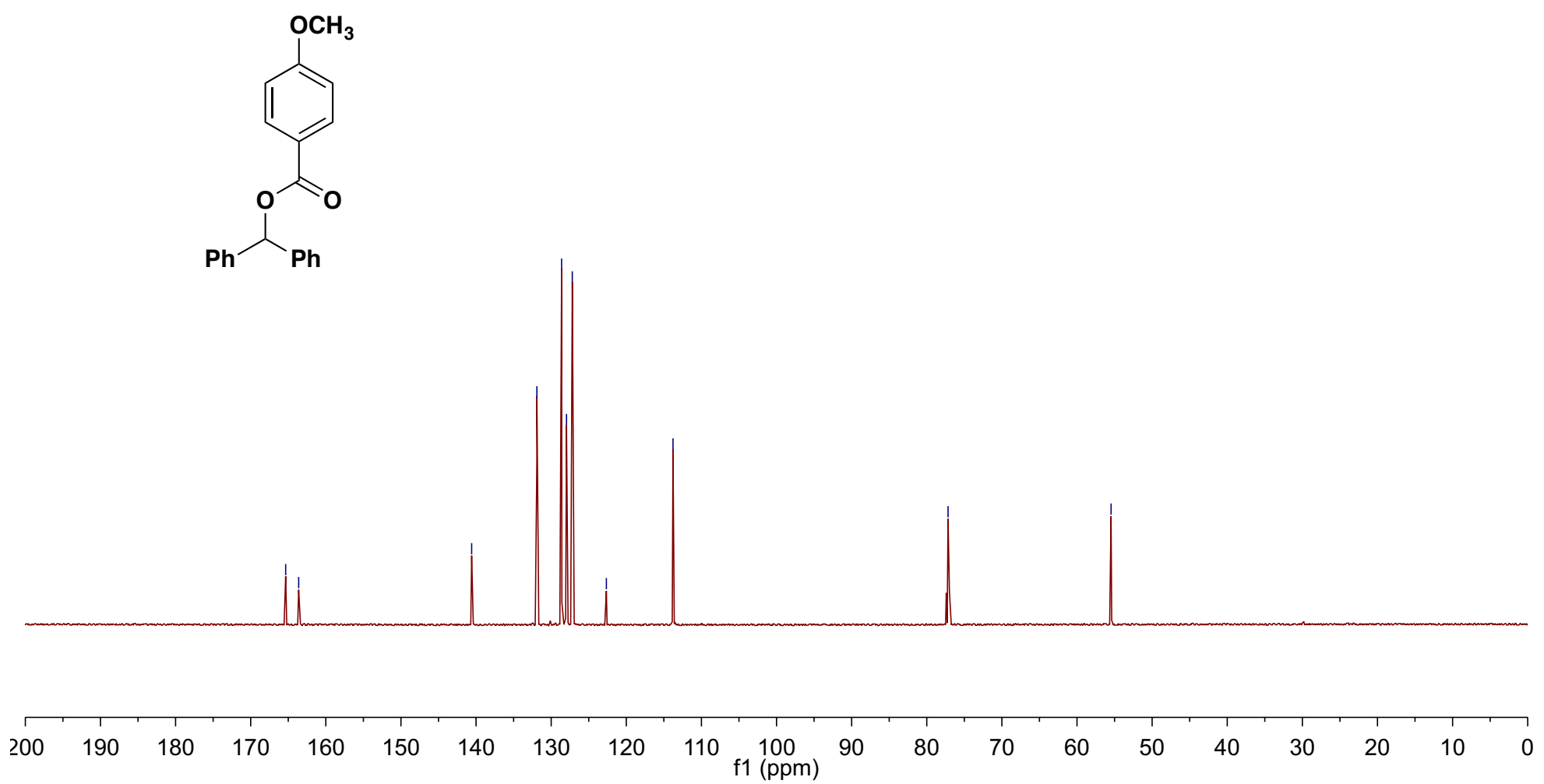




\section{benzhydryl 4-cyanobenzoate (3b) ${ }^{1} \mathrm{H}$ NMR (600 MHz, $\left.\mathrm{CDCl}_{3}\right)$ - Method A}

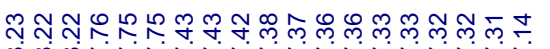

$\sqrt{n}$

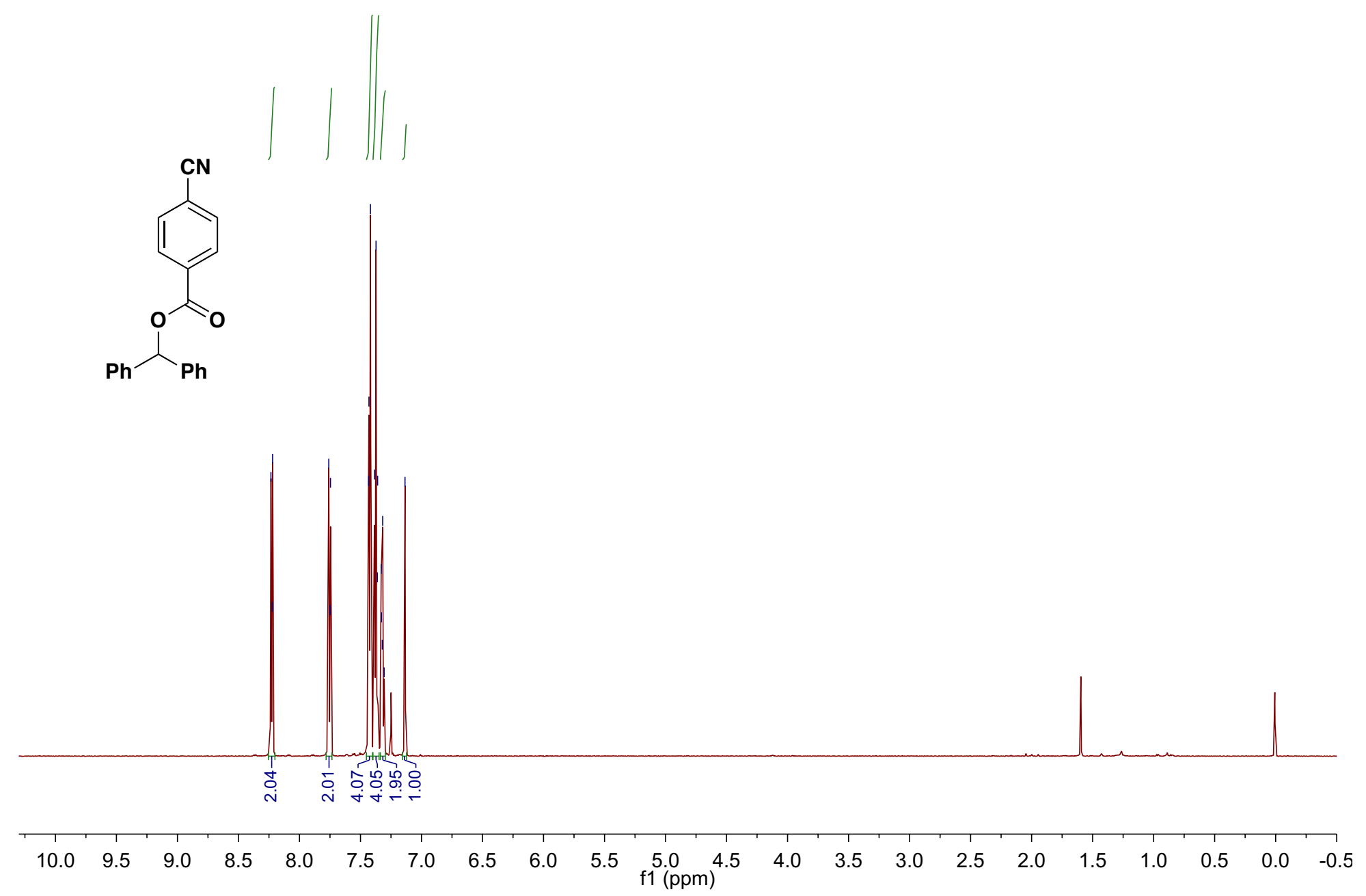


benzhydryl 4-cyanobenzoate (3b) ${ }^{13} \mathrm{C}$ NMR (151 MHz, $\left.\mathrm{CDCl}_{3}\right)-\operatorname{Method~A}$

年

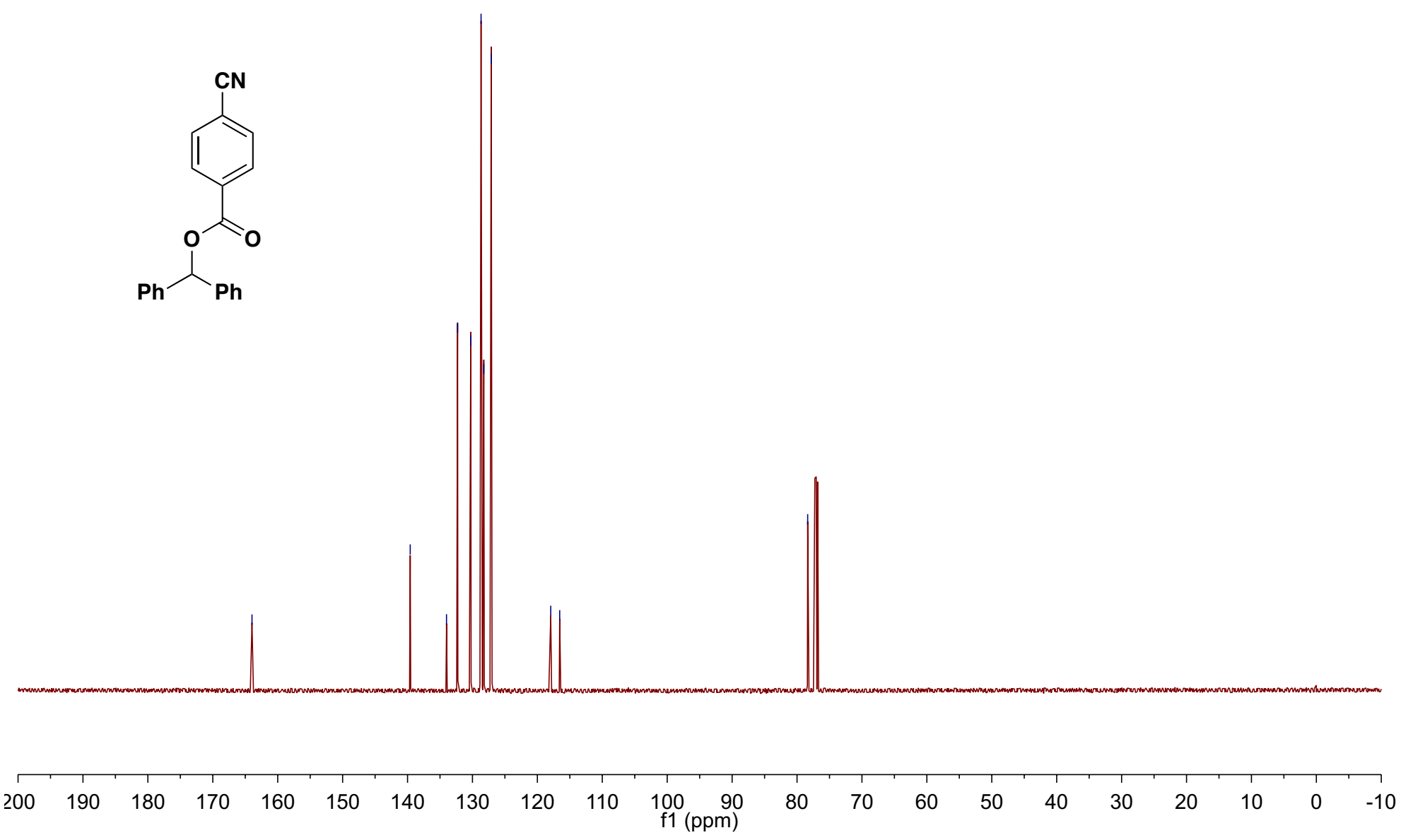


benzhydryl 4-hydroxybenzoate (3c) ${ }^{1} \mathrm{H}$ NMR (600 MHz, $\left.\mathrm{CDCl}_{3}\right)$ - Method A

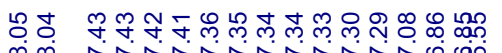

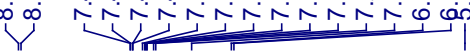

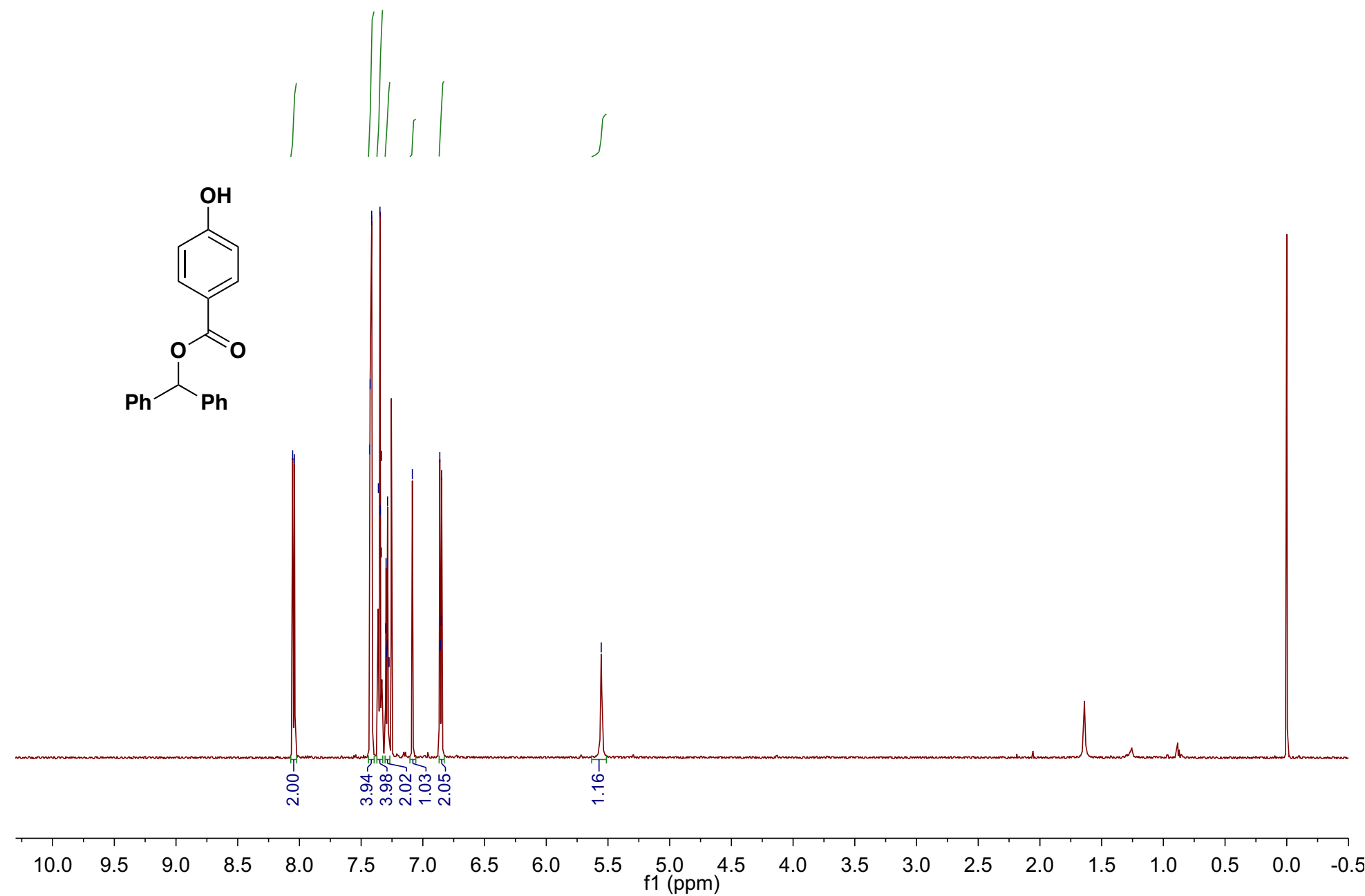


benzhydryl 4-hydroxybenzoate (3c) ${ }^{13} \mathrm{C}$ NMR (151 MHz, $\left.\mathrm{CDCl}_{3}\right)$ - Method A

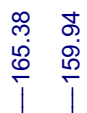
|
$\stackrel{\stackrel{2}{N}}{\stackrel{1}{*}}$

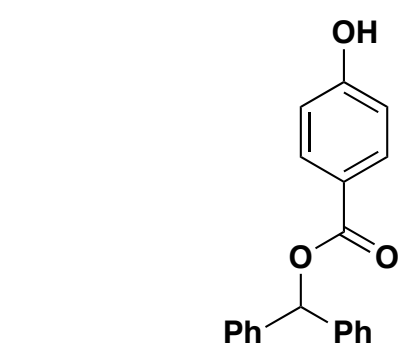

$\mathrm{OH}$

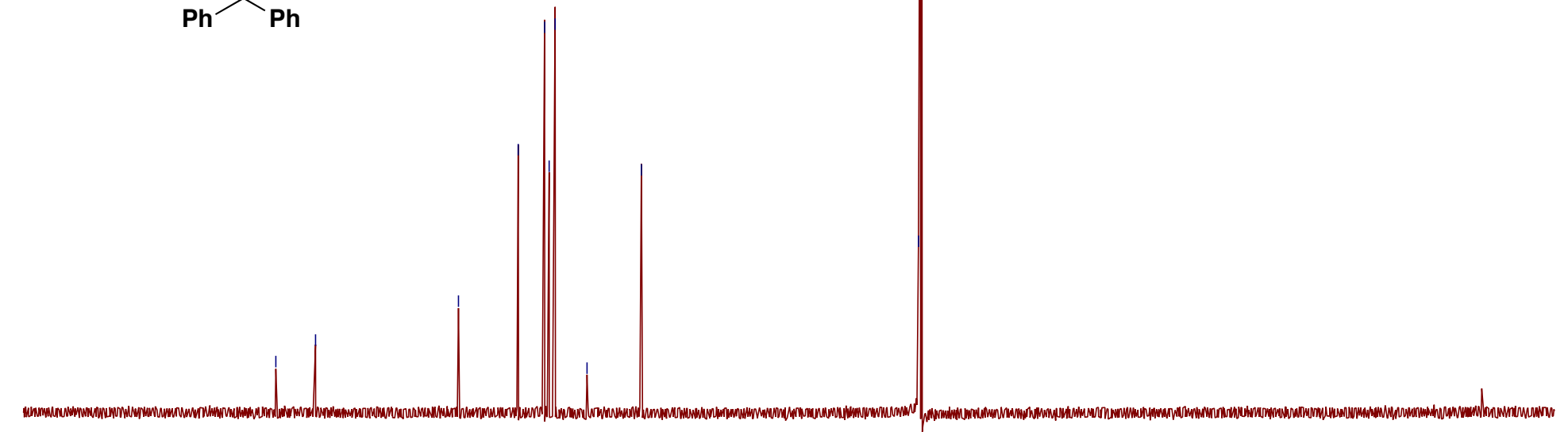


benzhydryl cinnamate (3d) ${ }^{1} \mathrm{H}$ NMR (600 MHz, $\left.\mathrm{CDCl}_{3}\right)-$ Method B

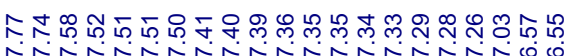

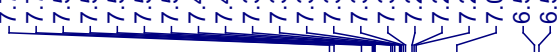

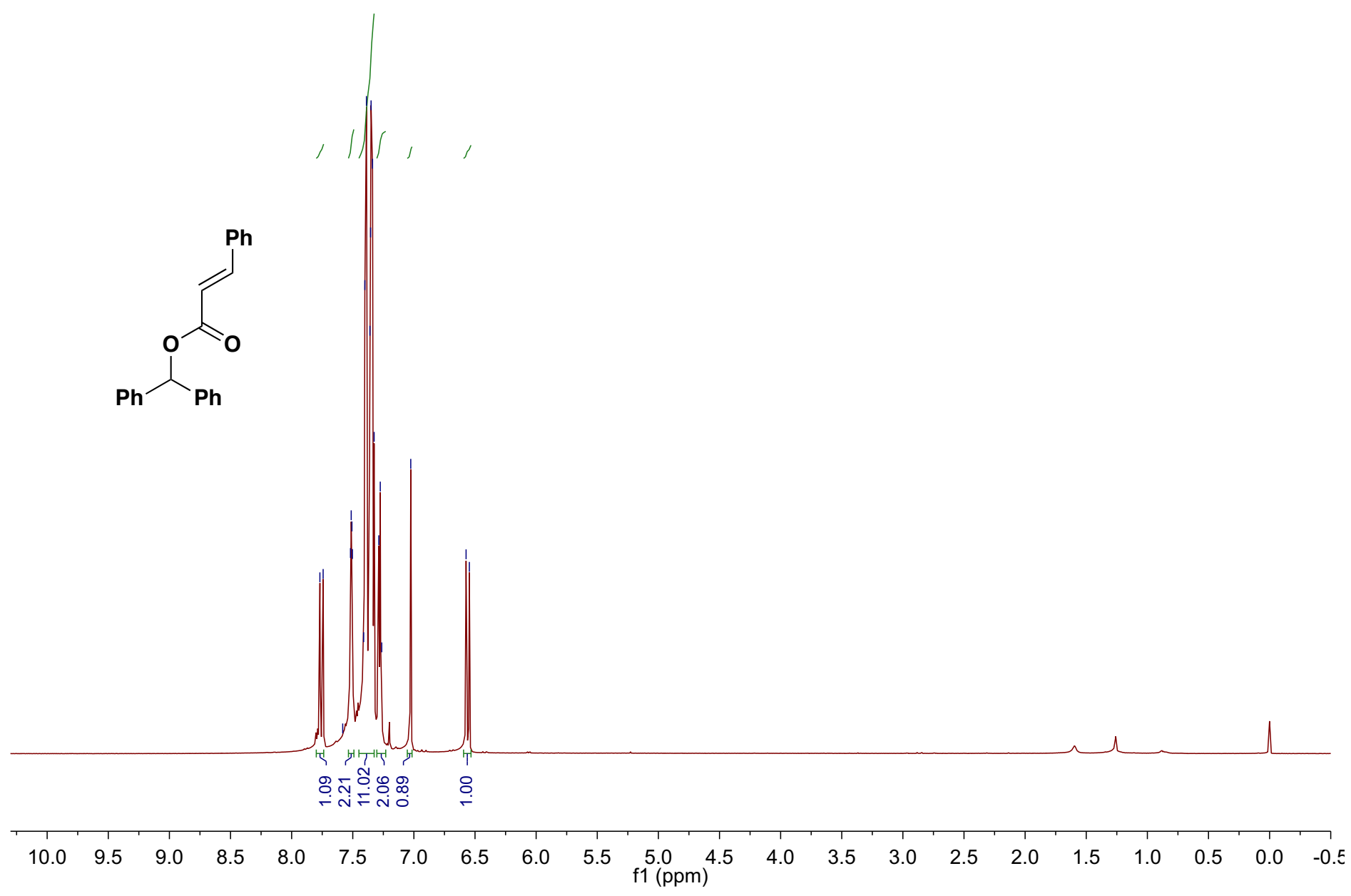


benzhydryl 3-phenylpropanoate (3e) ${ }^{1} \mathrm{H}$ NMR (600 $\mathrm{MHz}_{2} \mathrm{CDCl}_{3}$ ) - Method A

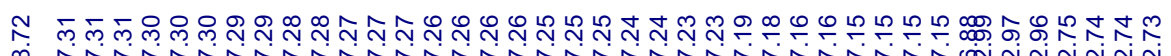

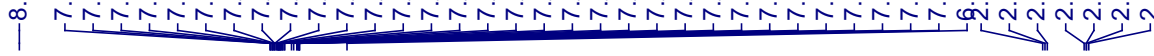

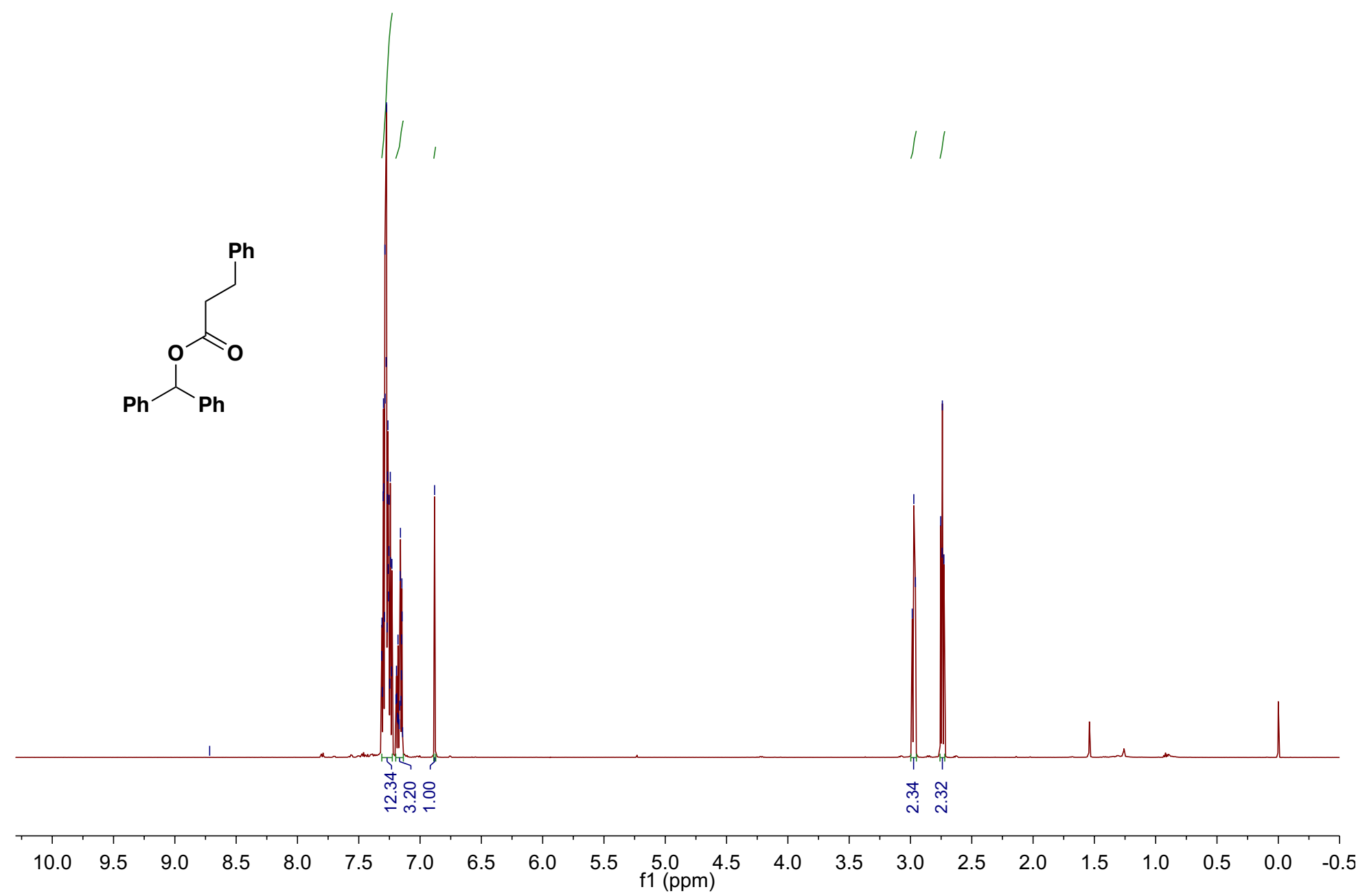


benzhydryl pivalate (3f) ${ }^{1} \mathrm{H}$ NMR (600 MHz, $\left.\mathrm{CDCl}_{3}\right)-$ Method A

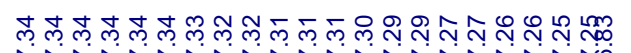

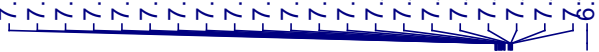
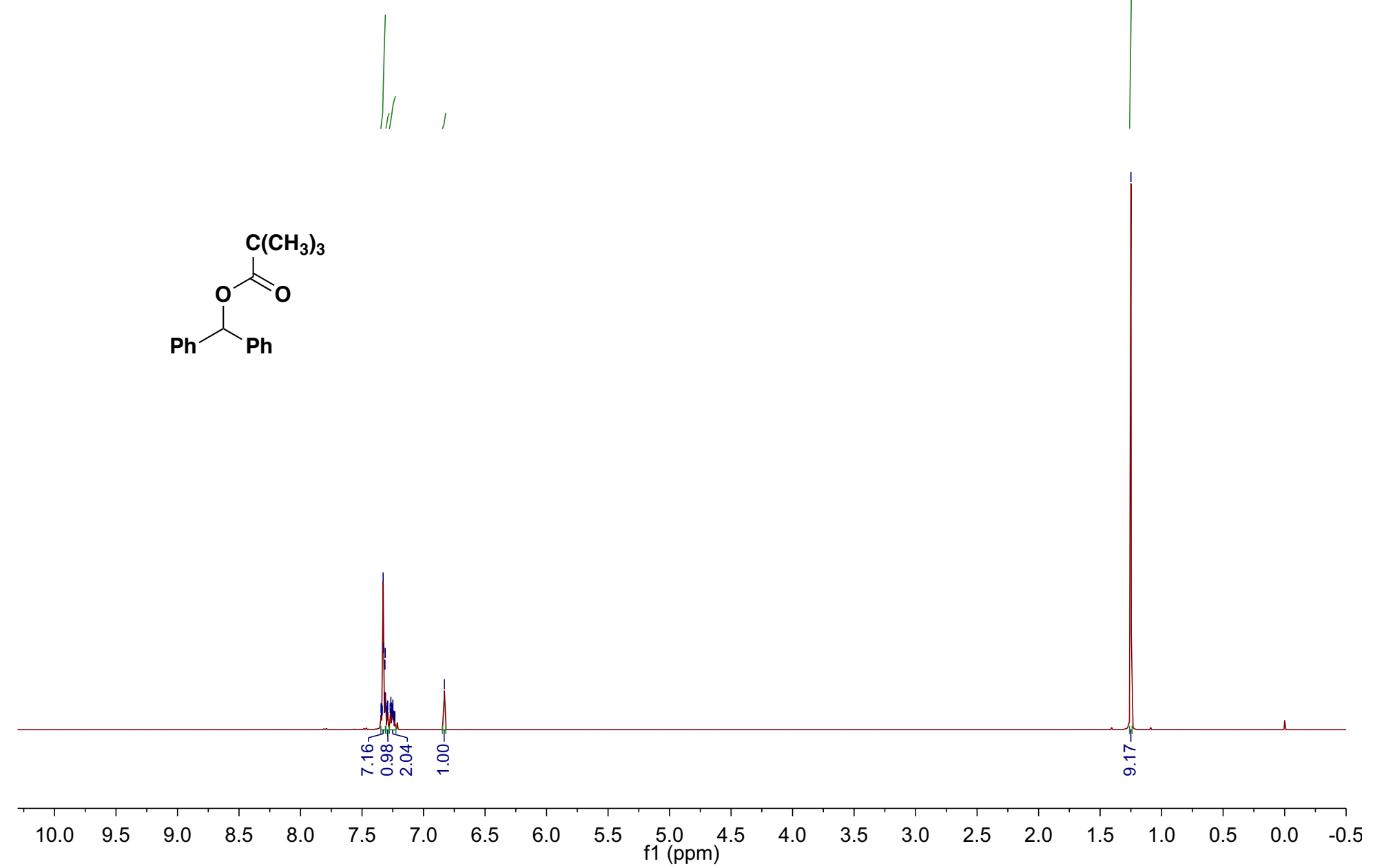
benzhydryl acetate (3g) ${ }^{1} \mathrm{H}$ NMR (600 MHz, $\left.\mathrm{CDCl}_{3}\right)$ - Method A

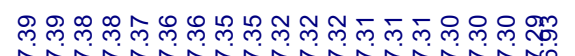

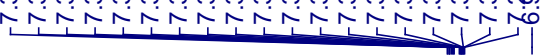
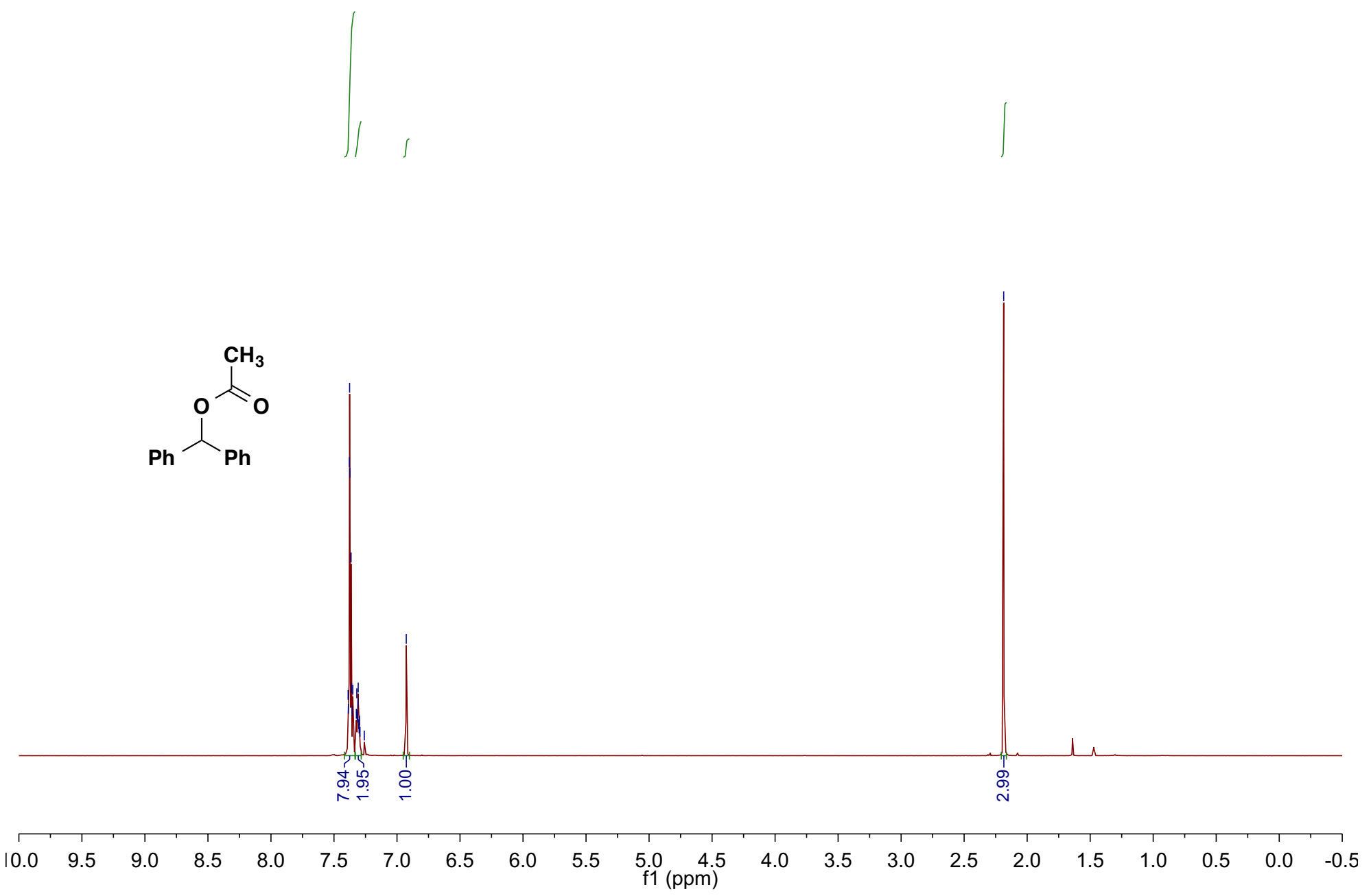


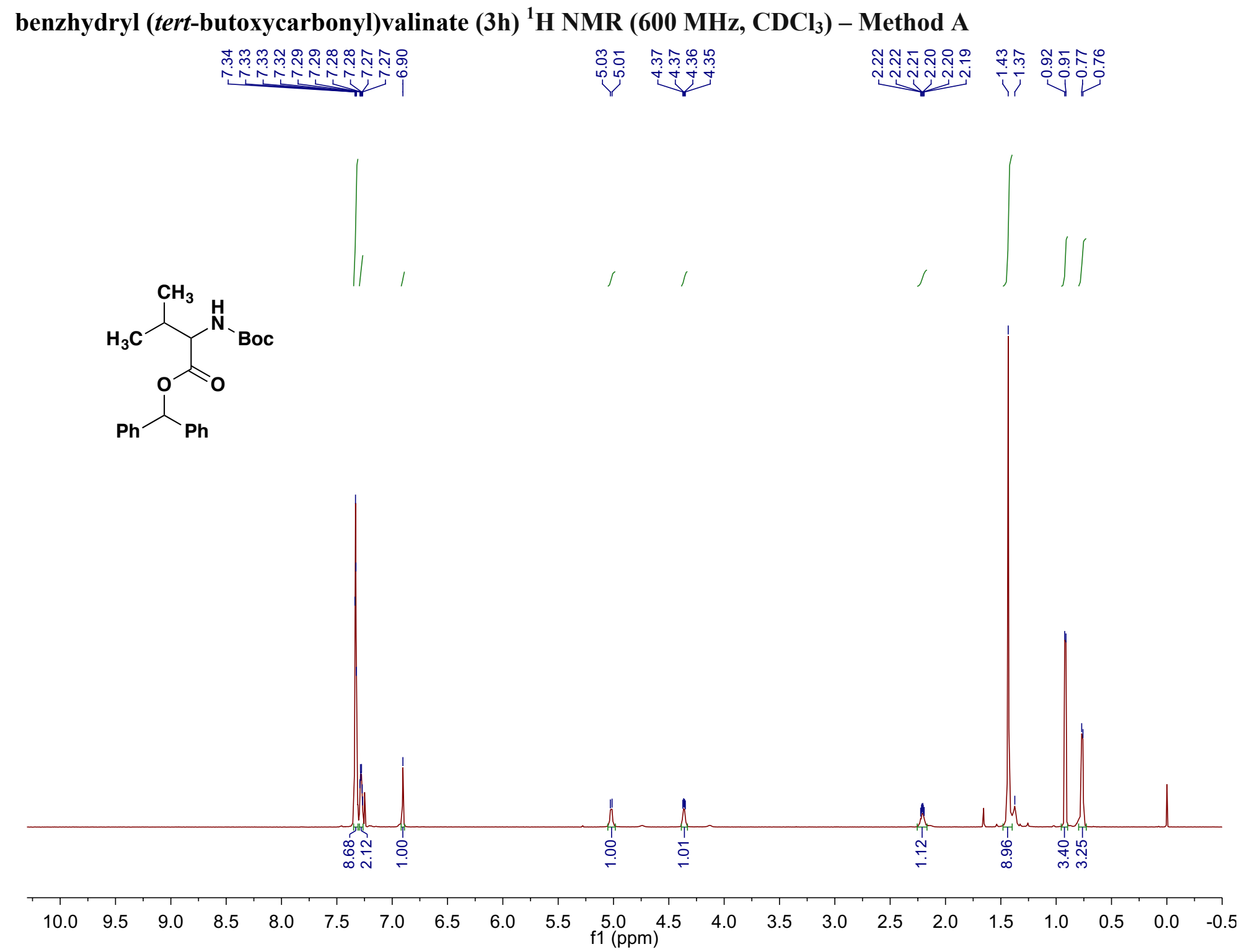


benzhydryl (tert-butoxycarbonyl)valinate $(3 \mathrm{~h}){ }^{13} \mathrm{C}$ NMR $\left(151 \mathrm{MHz}, \mathrm{CDCl}_{3}\right)-$ Method A

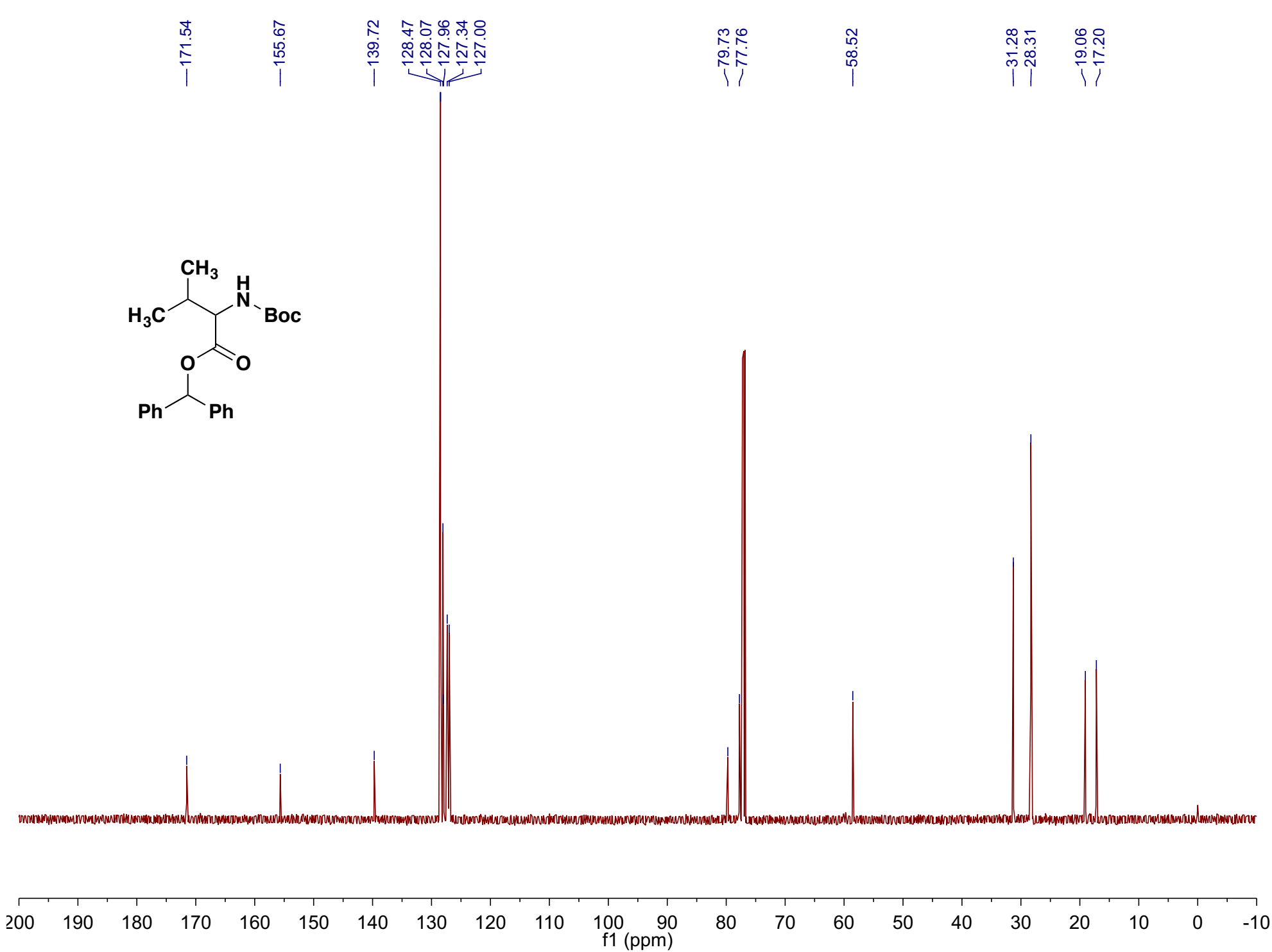


benzhydryl furan-3-carboxylate (3i) ${ }^{1} \mathrm{H}$ NMR (600 MHz, $\left.\mathrm{CDCl}_{3}\right)$

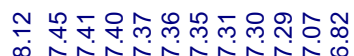

or

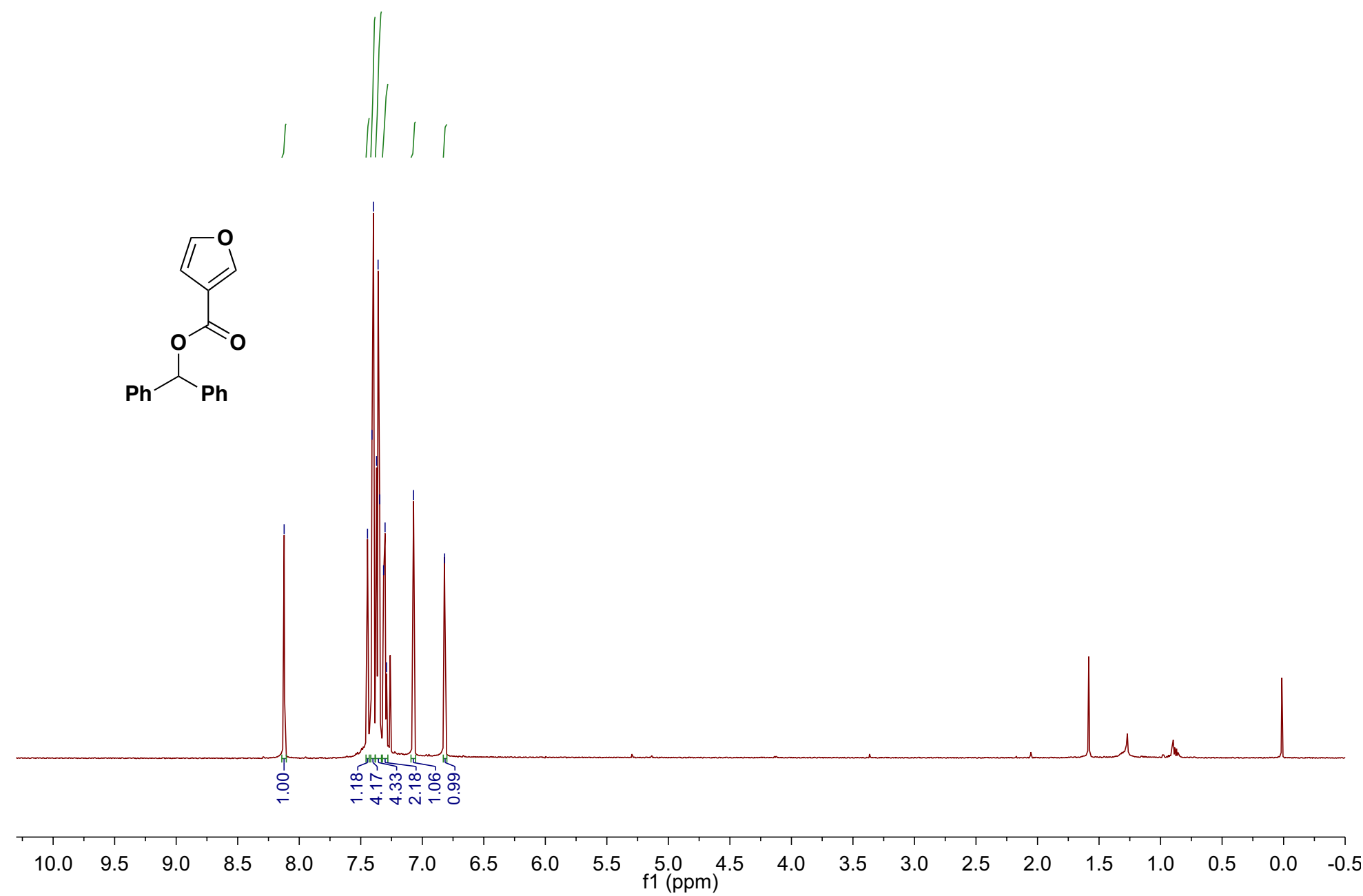


benzhydryl furan-3-carboxylate (3i) ${ }^{13} \mathrm{C}$ NMR (151 $\left.\mathrm{MHz}, \mathrm{CDCl}_{3}\right)-$ Method A
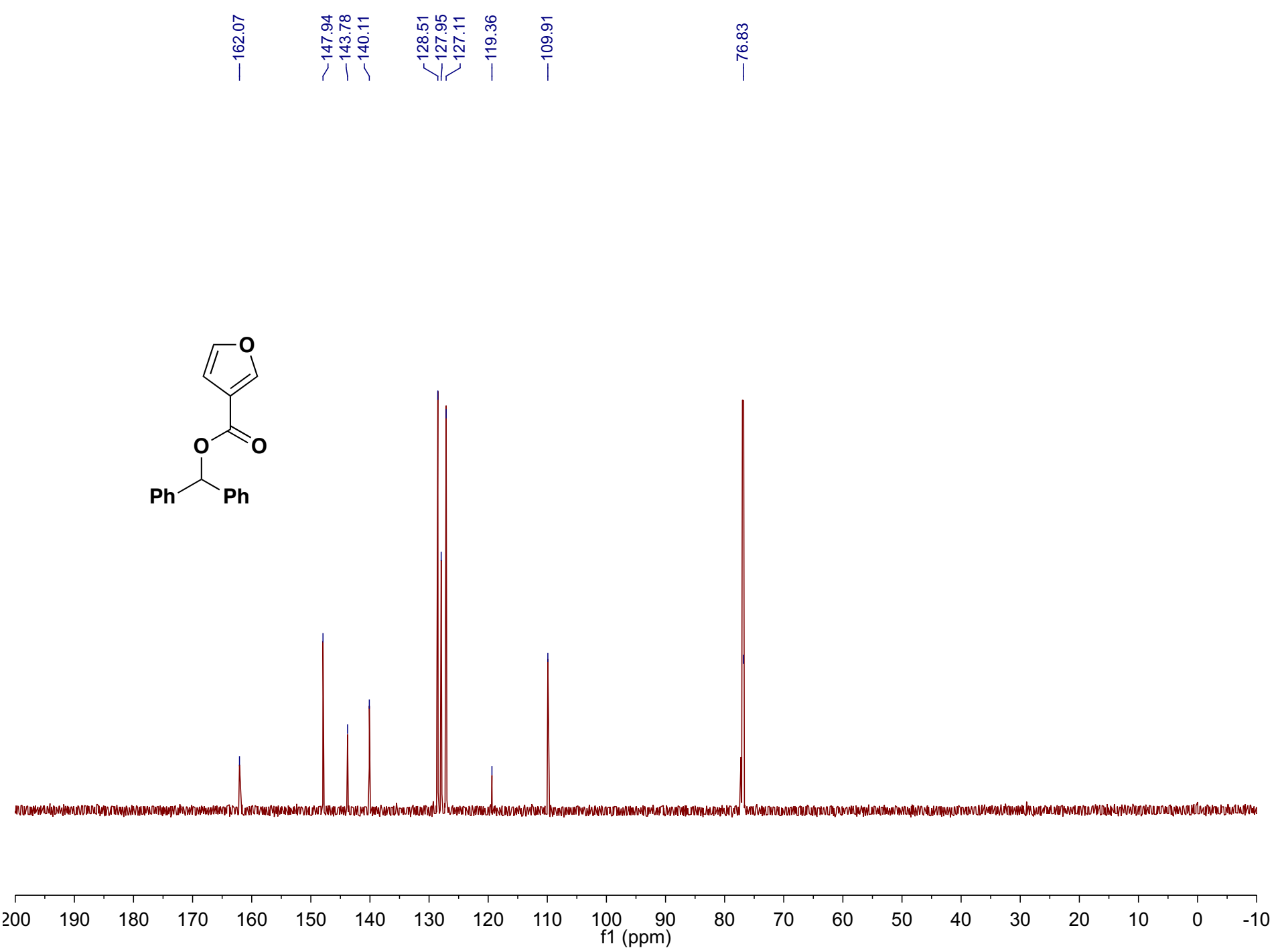
benzyl benzoate (5a) ${ }^{1} \mathrm{H}$ NMR (600 MHz, $\left.\mathrm{CDCl}_{3}\right)$ - Method A

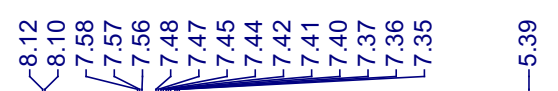
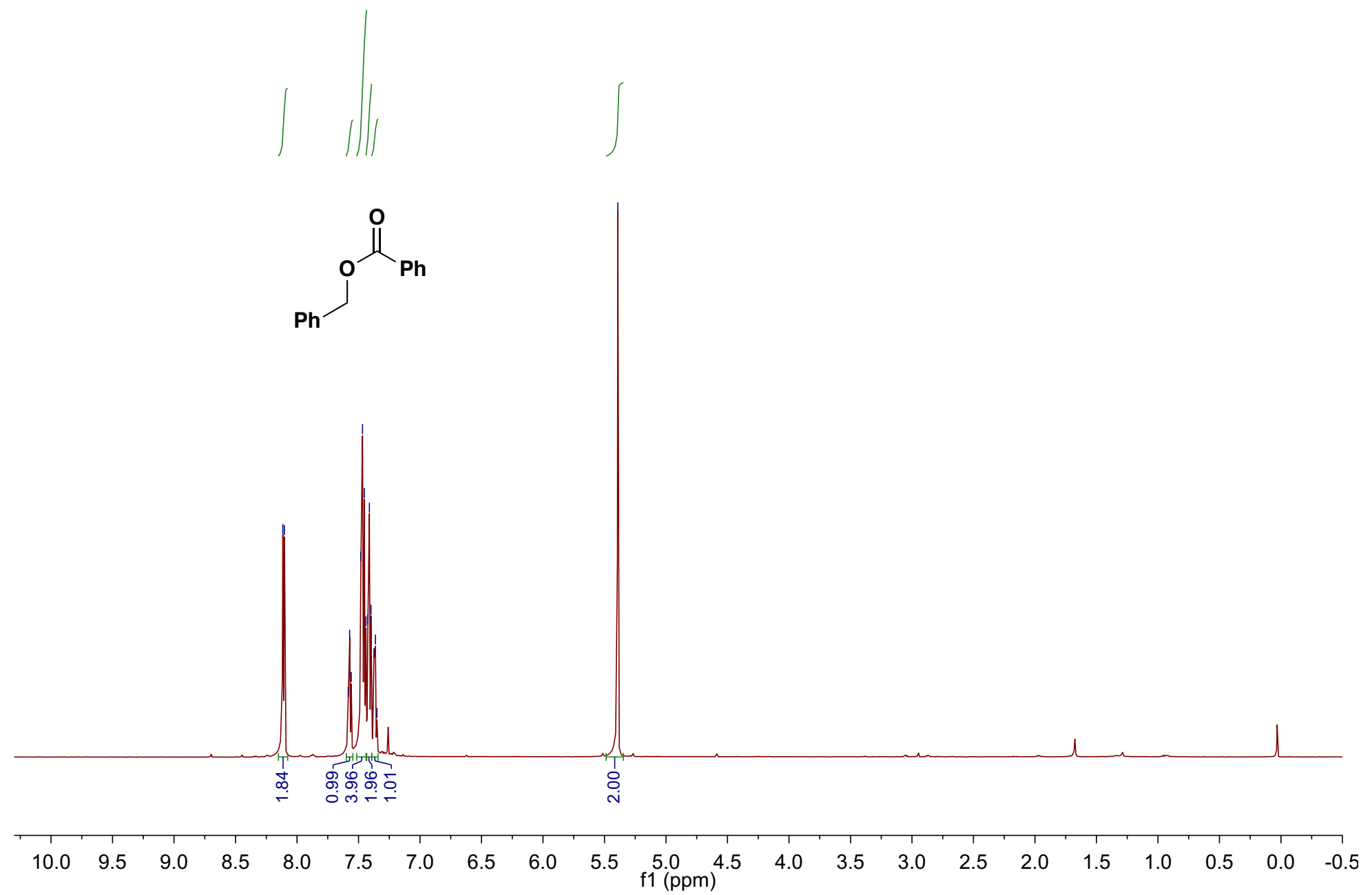
4-methoxybenzyl benzoate (5b) ${ }^{1} \mathrm{H}$ NMR (600 $\left.\mathrm{MHz}, \mathrm{CDCl}_{3}\right)$ - Method A

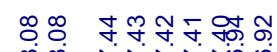

in

i̊

$\stackrel{\infty}{\infty}$
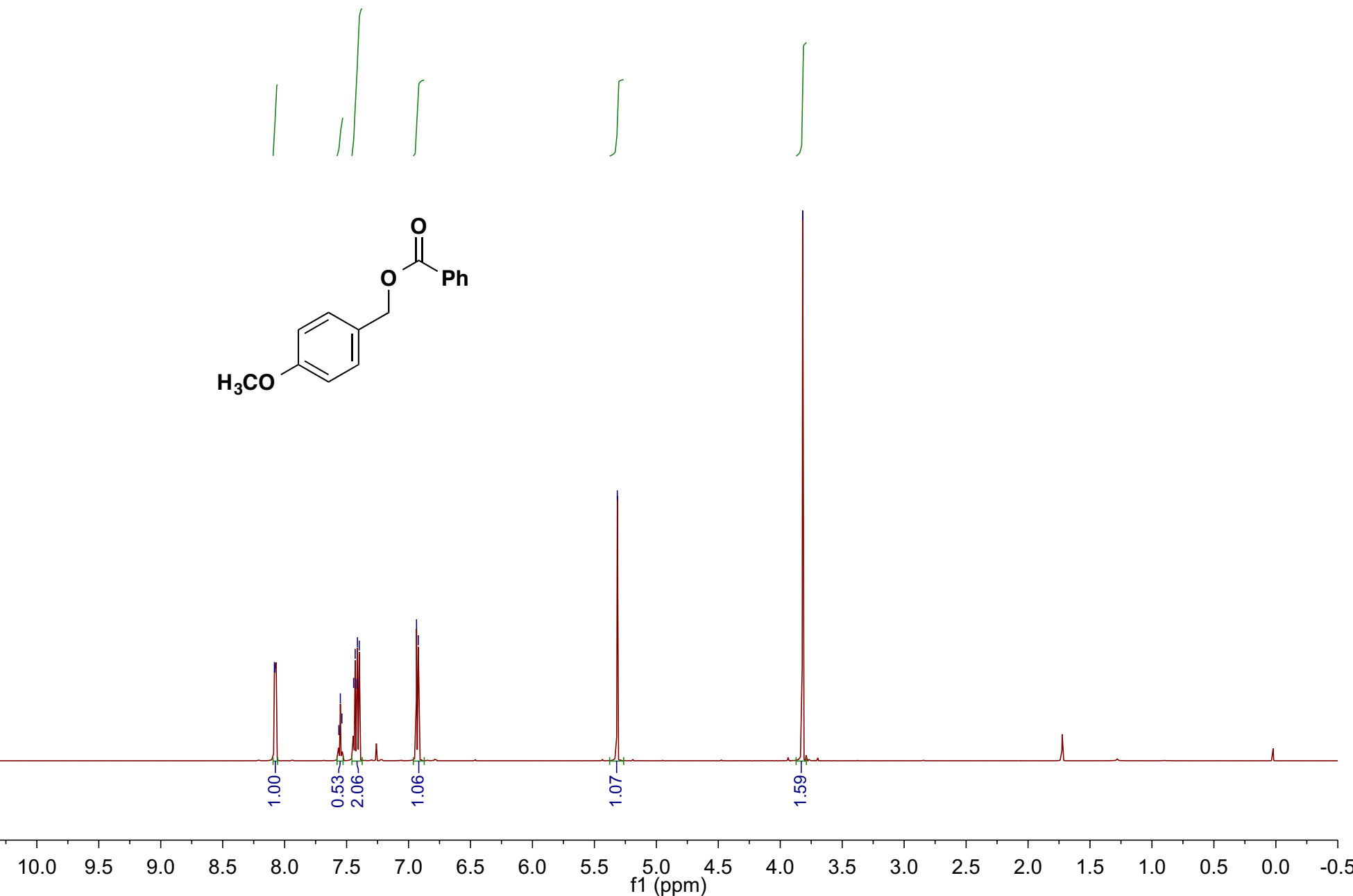
4-cyanobenzyl benzoate (5c) ${ }^{1} \mathrm{H}$ NMR (600 $\left.\mathrm{MHz}, \mathrm{CDCl}_{3}\right)$ - Method B

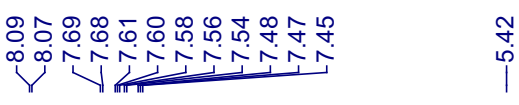

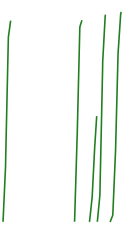

官

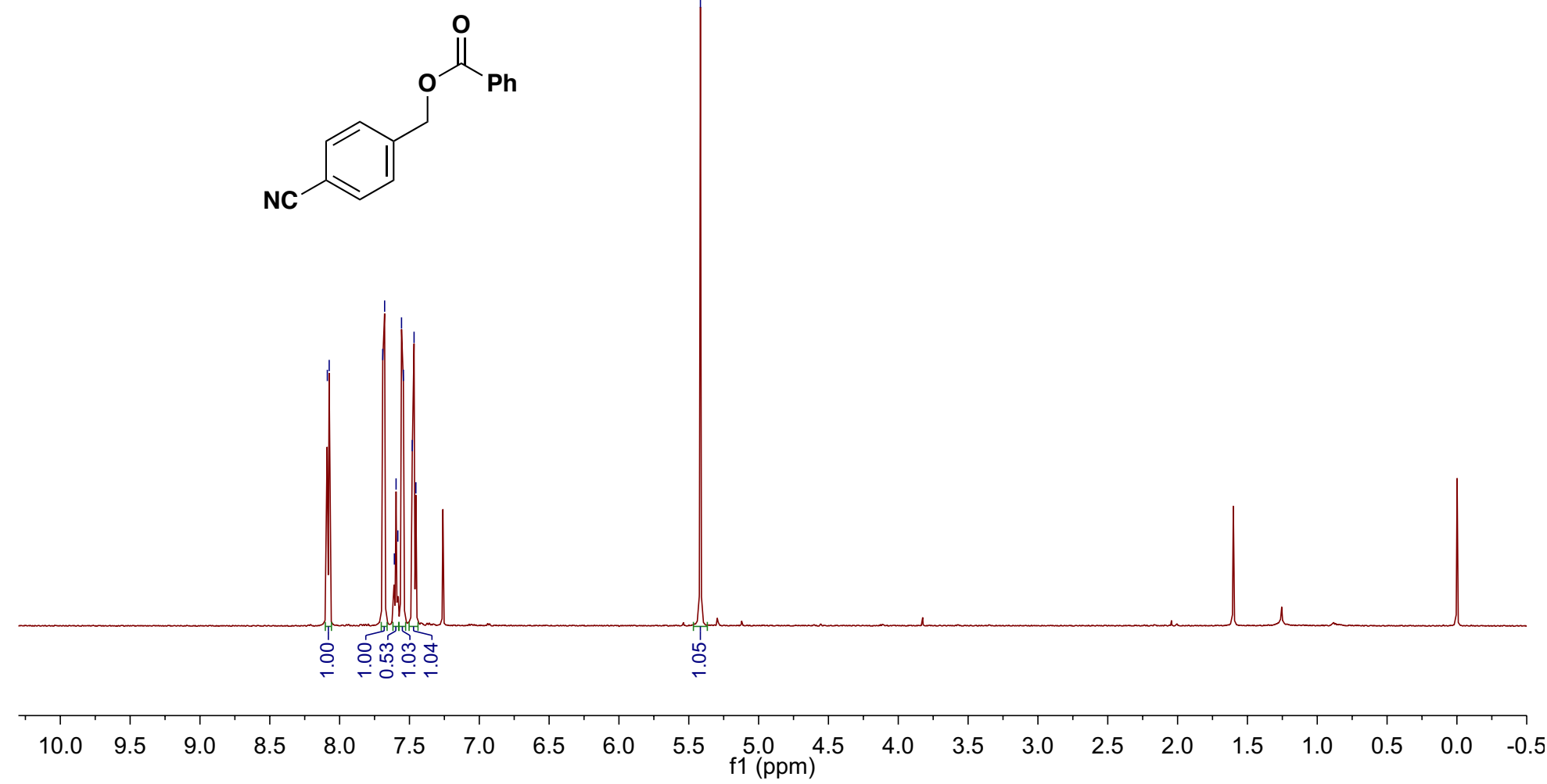


2,4,6-trimethylbenzyl benzoate (5d) ${ }^{1} \mathrm{H}$ NMR (600 $\left.\mathrm{MHz}, \mathrm{CDCl}_{3}\right)-\operatorname{Method~A}$
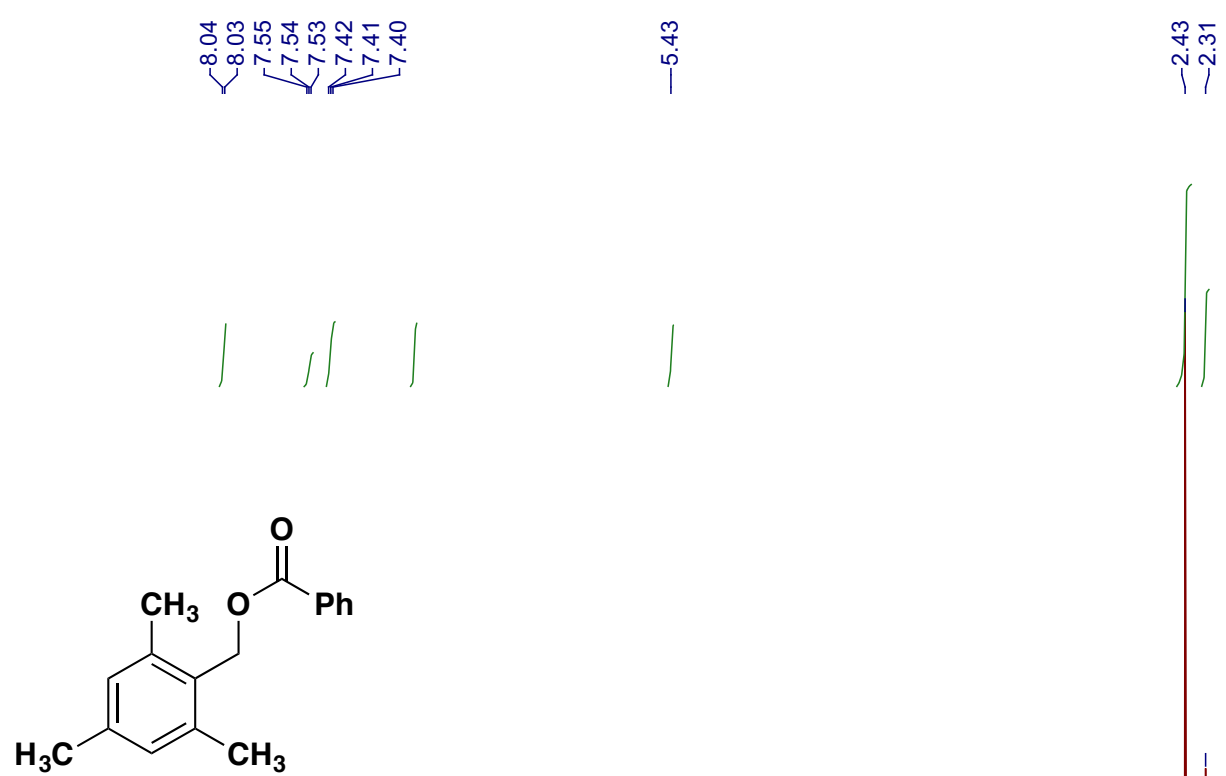

$\stackrel{m}{i} \stackrel{m}{j}_{1}^{-1}$

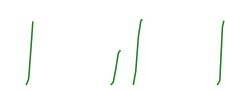

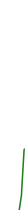

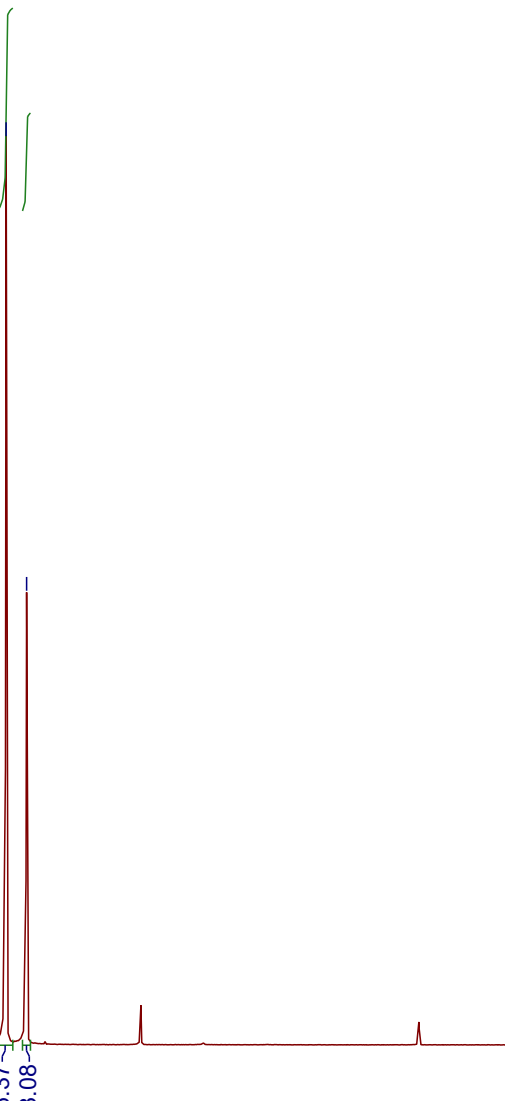

10.09

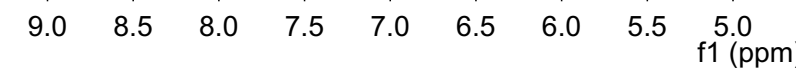

f1 5.0 ppm)

$4.0 \quad 3.5$

$3.0 \quad 2.5$

$2.0 \quad 1.5$ 
2,4,6-trimethylbenzyl benzoate (5d) ${ }^{13} \mathrm{C}$ NMR (151 MHz, $\left.\mathrm{CDCl}_{3}\right)$ - Method A
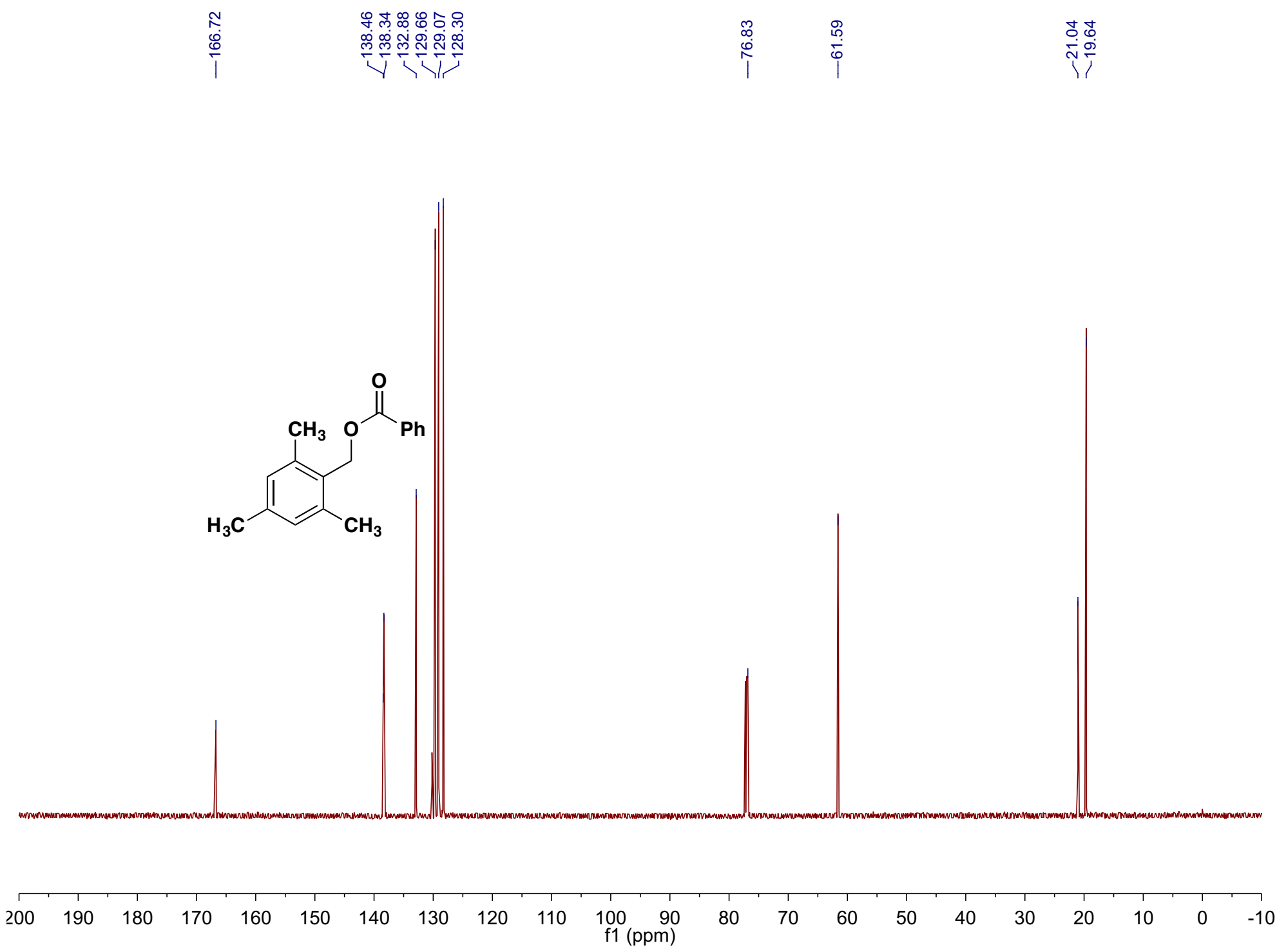
1-phenylpropyl benzoate (5e) ${ }^{1} \mathrm{H}$ NMR (600 MHz, CDCl 3$)-$ Method B

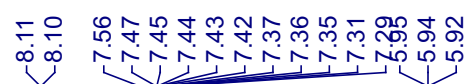

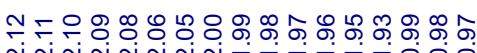

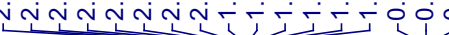
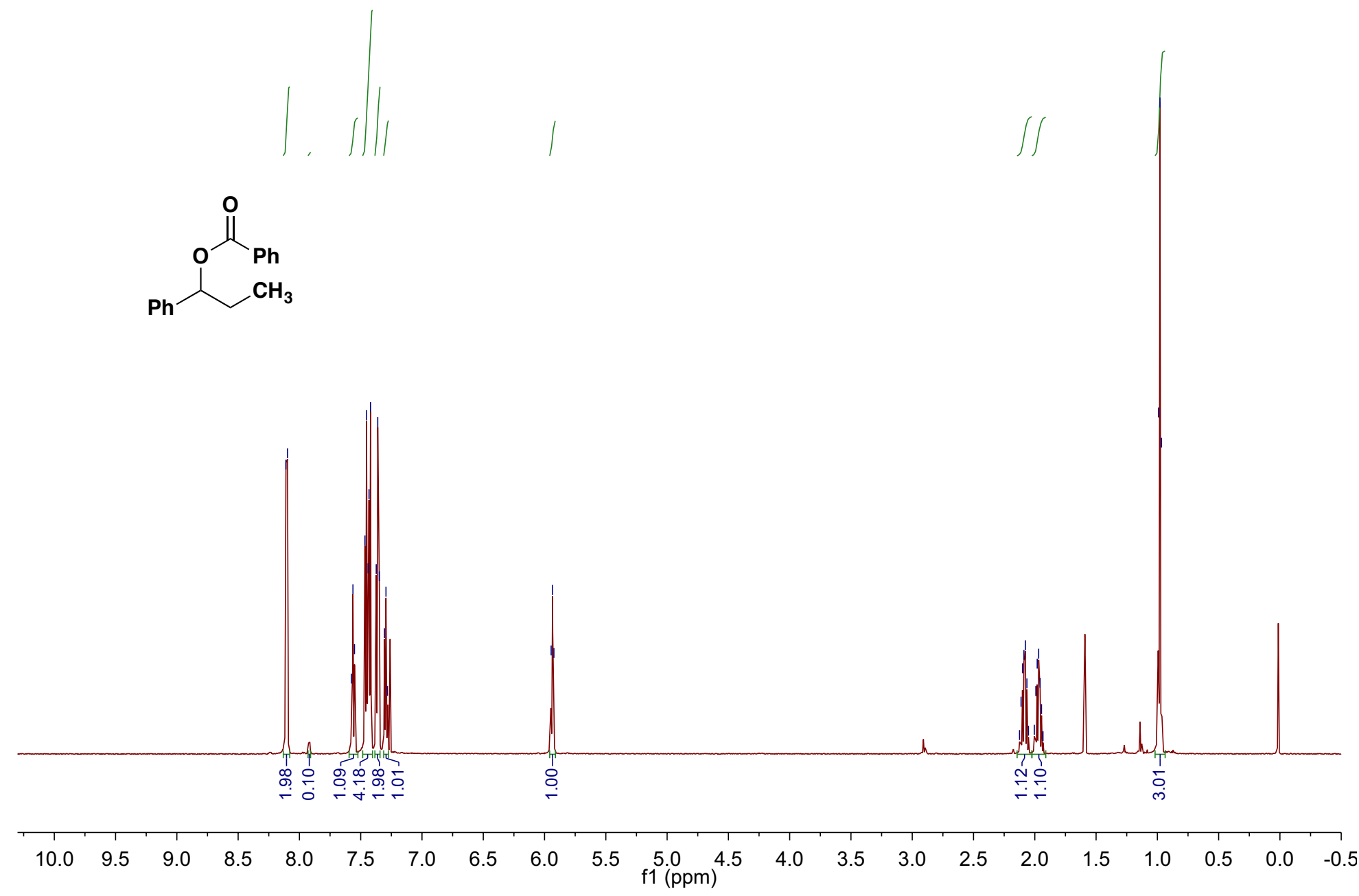
1-phenylethyl benzoate (5f) ${ }^{1} \mathrm{H}$ NMR (600 $\left.\mathrm{MHz}, \mathrm{CDCl}_{3}\right)-$ Method A

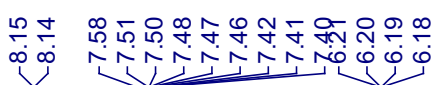
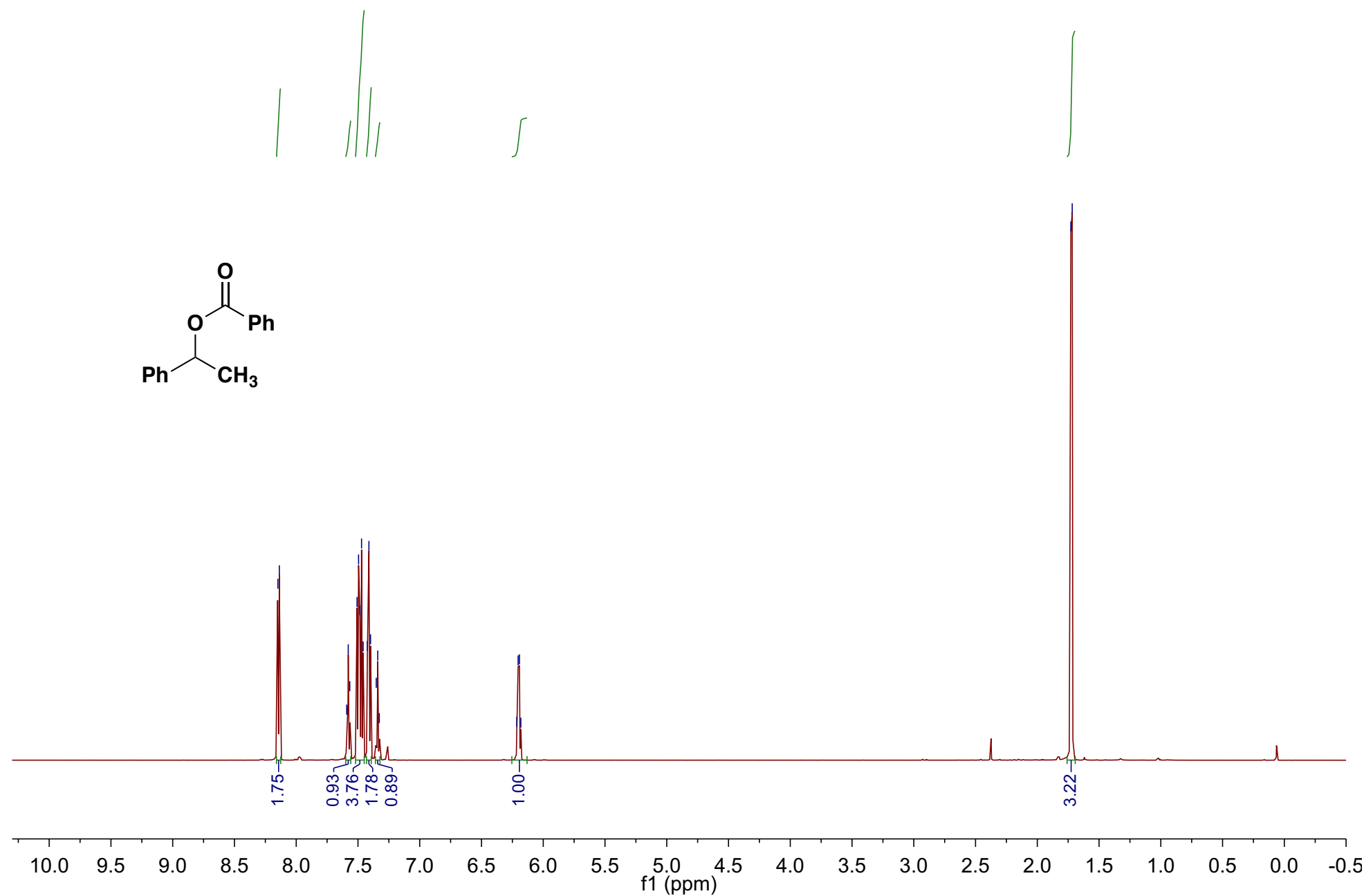
1,2,3,4-tetrahydronaphthalen-1-yl benzoate (5g) ${ }^{1} \mathrm{H}$ NMR (600 $\left.\mathrm{MHz}, \mathrm{CDCl}_{3}\right)$ - $\mathrm{Method} \mathrm{B}$

。

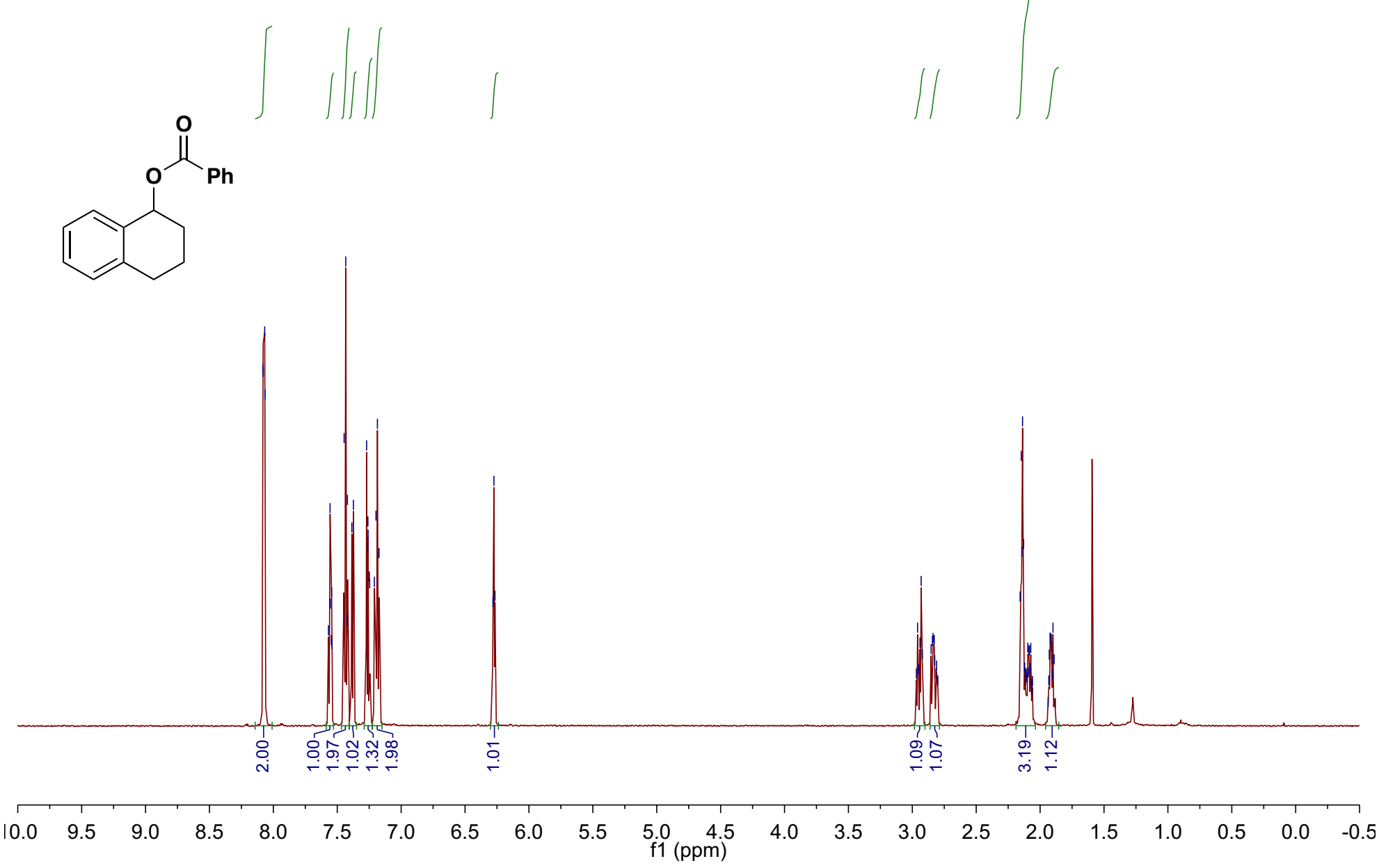


benzyl cyclohexanecarboxylate (5h) ${ }^{1} \mathrm{H}$ NMR (600 $\left.\mathrm{MHz} \mathrm{CDCl}_{3}\right)-$ Method A
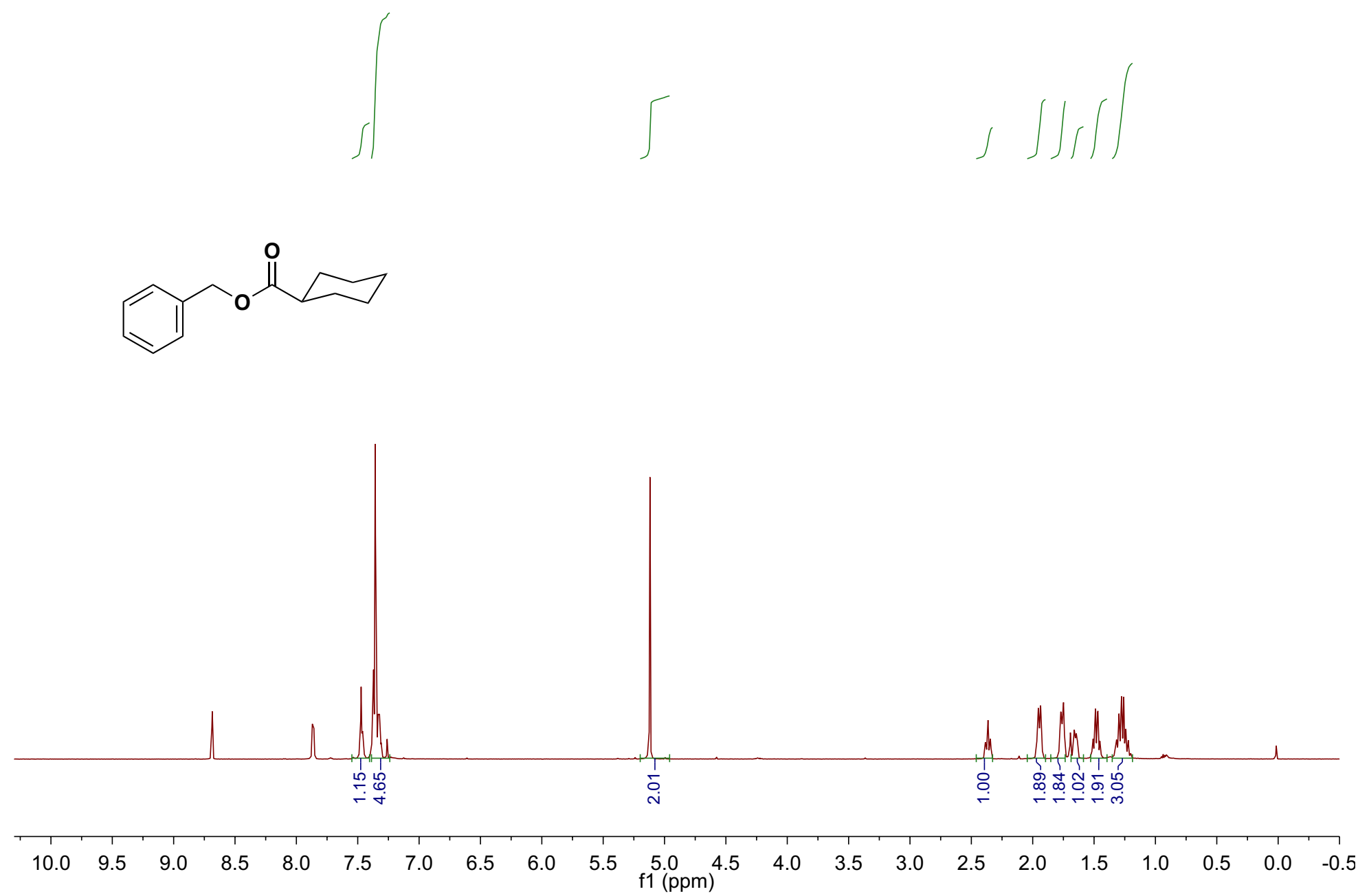
4-methoxybenzyl cyclohexanecarboxylate (5i) ${ }^{1} \mathrm{H}$ NMR (600 $\left.\mathrm{MHz}, \mathrm{CDCl}_{3}\right)-\mathrm{Method}^{\mathrm{A}}$
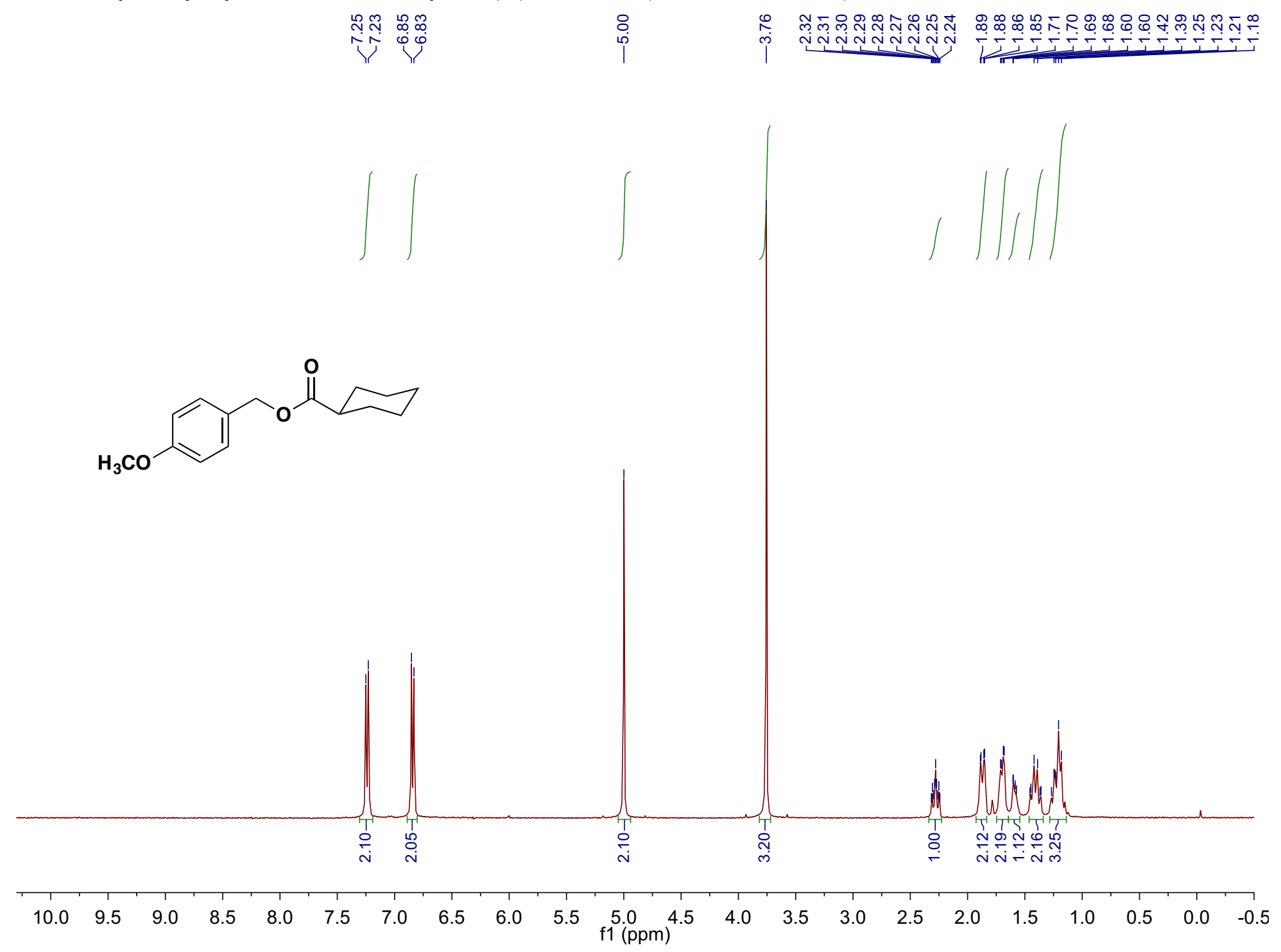
References

(1) Ohishi, T.; Yamada, J.; Inui, Y.; Sakaguchi, T.; Yamashita, M. J. Org. Chem. 1994, 59, 7521-7522.

(2) Kleine, T.; Frohlich, R.; Wibbeling, B.; Wurthwein, E. U. J. Org. Chem. 2011, 76, 45914599 .

(3) Lee, K.-J.; Kim, J. L.; Hong, M. K.; Lee, J. Y. J. Heterocycl. Chem. 1999, 36, 1235-1240.

(4) Poh, J. S.; Tran, D. N.; Battilocchio, C.; Hawkins, J. M.; Ley, S. V. Angew. Chem., Int. Ed. 2015, 54, 7920-7923.

(5) Zhang, C.; Feng, P.; Jiao, N. J. Am. Chem. Soc. 2013, 135, 15257-15262.

(6) Magens, S.; Plietker, B. J. Org. Chem. 2010, 75, 3715-3721.

(7) Chen, C. T.; Munot, Y. S. J. Org. Chem. 2005, 70, 8625-8627.

(8) Strazzolini, P.; Giumanini, A. G.; Verardo, G. Tetrahedron 1994, 50, 217-254.

(9) Lim, S.; Ji, M.; Wang, X.; Lee, C.; Jang, H.-Y. Eur. J. Org. Chem. 2015, 2015, 591-595.

(10) Liu, H.; Shi, G.; Pan, S.; Jiang, Y.; Zhang, Y. Org. Lett. 2013, 15, 4098-4101.

(11) Liu, C.; Tang, S.; Zheng, L.; Liu, D.; Zhang, H.; Lei, A. Angew. Chem., Int. Ed. 2012, 51, $5662-5666$.

(12) Wommack, A. J.; Kingsbury, J. S. J. Org. Chem. 2013, 78, 10573-10587.

(13) Liu, Z.; Ma, Q.; Liu, Y.; Wang, Q. Org. Lett. 2014, 16, 236-239. 